\title{
Platinum-Catalyzed Intramolecular Hydroamination of Unactivated Olefins with Secondary Alkyl Amines
}

\author{
Christopher F. Bender and Ross A. Widenhoefer* \\ P. M. Gross Chemical Laboratory \\ Duke University
}

Durham, North Carolina 27708-0346

\section{Supporting Information}

Expanded discussion of the formation and characterization of platinum intermediates

trans $\mathbf{- 5 , 6}$, and trans $\mathbf{- 8}$, experimental procedures, analytical and spectroscopic data, and copies of NMR spectra for selected compounds (63 pages). 


\section{Experimental}

General Methods. Reactions were performed under a nitrogen atmosphere employing standard Schlenk and/or drybox techniques unless specified otherwise. NMR were obtained on a Varian spectrometer operating at $400 \mathrm{MHz}$ for ${ }^{1} \mathrm{H}, 202 \mathrm{MHz}$ for ${ }^{31} \mathrm{P}$, and $100 \mathrm{MHz}$ for ${ }^{13} \mathrm{C}$ in $\mathrm{CDCl}_{3}$ at $25^{\circ} \mathrm{C}$ unless stated otherwise. IR spectra were obtained on a Nicolet Avatar 360-FT IR spectrometer. Gas chromatography was performed on a HP 5890 gas chromatograph equipped with a $25 \mathrm{~m}$ polydimethylsiloxane capillary column. Column chromatography was performed employing 230-450 mesh silica gel (Sorbent Technologies) unless noted otherwise; alternatively, chromatography employed 150 mesh activated aluminum oxide, neutral, Brockmann I (Aldrich). Thin layer chromatography (TLC) was performed on silica gel $60 \mathrm{~F}_{254}$. All compounds were isolated as colorless oils unless noted otherwise. Elemental analyses were performed by Complete Analysis Laboratories (Parsippany, NJ).

1,4-Dioxane (anhydrous, Acros), triphenylphosphine (Fluka), and $\left[\mathrm{PtCl}_{2}\left(\mathrm{C}_{2} \mathrm{H}_{4}\right)\right]_{2}$ (2) (Strem) were used as received. Tetrahydrofuran (THF) and diethyl ether were distilled from $\mathrm{Na}^{0}$ /benzoquinone under $\mathrm{N}_{2}$, dichloromethane was distilled from $\mathrm{CaH}_{2}$ under $\mathrm{N}_{2}$, and dioxane- $d_{8}$ was distilled from $\mathrm{Na} / \mathrm{K}$ alloy under vacuum. $\left[\mathrm{PtCl}_{2}\left(\mathrm{PPh}_{3}\right)\right]_{2}$ (4) was synthesized employing a published procedure. ${ }^{\mathrm{S} 1}$ All other reagents were purchased from major chemical suppliers and were used as received. 


\section{Synthesis of Amino Olefins}

Benzyl(2,2-diphenyl-4-pentenyl)amine (1). Compound 1 was synthesized in $58 \%$ overall yield in three steps from diphenylacetonitrile (Scheme S1).
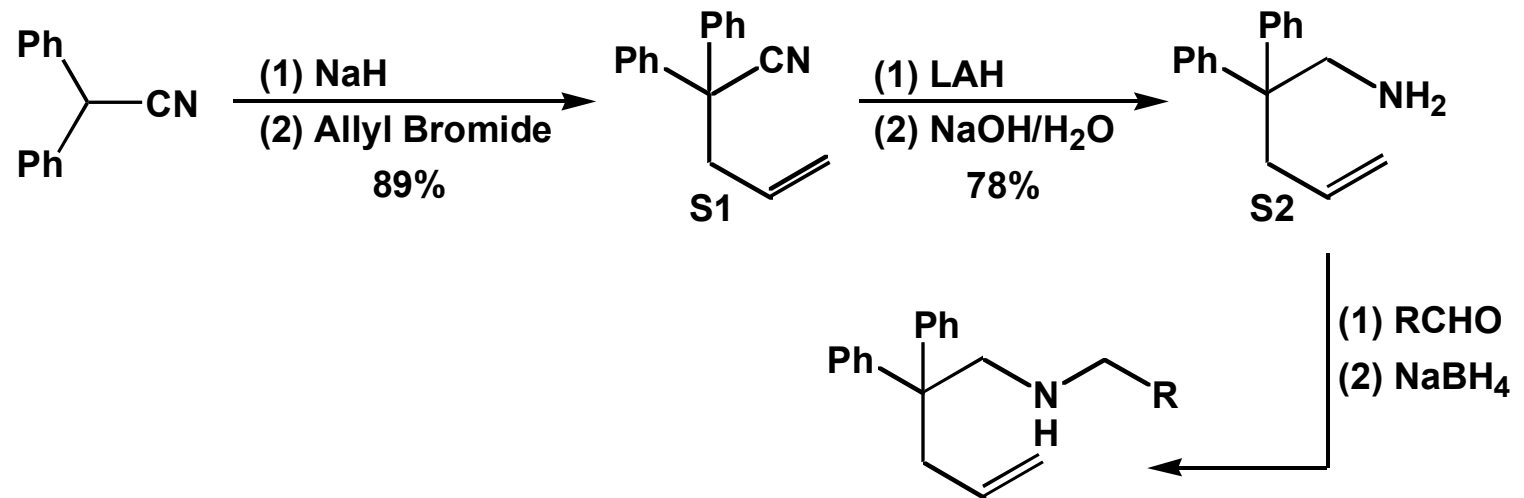

(1) $\mathrm{R}=\mathrm{Ph}(84 \%)$

(S3) $\mathrm{R}=4-\mathrm{C}_{6} \mathrm{H}_{4} \mathrm{Br}(86 \%)$

(S4) $\mathrm{R}=4-\mathrm{C}_{6} \mathrm{H}_{4} \mathrm{CN}(73 \%)$

(S5) $\mathrm{R}=4-\mathrm{C}_{6} \mathrm{H}_{4} \mathrm{NO}_{2}(57 \%)$

(S6) $\mathrm{R}=4-\mathrm{C}_{6} \mathrm{H}_{4} \mathrm{CO}_{2} \mathrm{Me}(86 \%)$

(S7) $\mathrm{R}=3,4-\mathrm{C}_{6} \mathrm{H}_{3}\left(-\mathrm{OCH}_{2} \mathrm{O}-\right)(70 \%)$

\section{Scheme S1}

A solution of diphenylacetonitrile $(19.3 \mathrm{~g}, 100 \mathrm{mmol})$ in DMF $(30 \mathrm{~mL})$ was added slowly to a suspension of $\mathrm{NaH}(2.52 \mathrm{~g}, 105 \mathrm{mmol})$ in $\mathrm{DMF}(100 \mathrm{~mL})$ and the resulting mixture was stirred at room temperature for $1 \mathrm{~h}$. The resulting bright yellow suspension was cooled to $0{ }^{\circ} \mathrm{C}$, treated with allyl bromide $(13.3 \mathrm{~g}, 110 \mathrm{mmol})$, and warmed to room temperature overnight with stirring. The resulting solution was poured into an ice/water mixture $(300 \mathrm{~mL})$ and extracted with benzene $(3 \times 100 \mathrm{~mL})$. The combined benzene extracts were washed with water $(2 \times 50 \mathrm{~mL})$, dried $\left(\mathrm{MgSO}_{4}\right)$, and concentrated to give 
2,2-diphenyl-4-pentenenitrile (S1) (20.7 g, 89\%), which was used in the subsequent step without further purification.

A suspension of $\mathrm{LiAlH}_{4}(12.4 \mathrm{~g}, 327 \mathrm{mmol})$ in ether $(130 \mathrm{~mL})$ was treated with S1 $(19.2 \mathrm{~g}, 82 \mathrm{mmol})$ at $0{ }^{\circ} \mathrm{C}$ and then warmed slowly to room temperature and stirred overnight. The resulting suspension was cooled to $0{ }^{\circ} \mathrm{C}$ and quenched by slow addition of $6 \mathrm{M} \mathrm{NaOH}(200 \mathrm{~mL})$. The resulting mixture was extracted with ether $(4 \times 100 \mathrm{~mL})$ and the combined ether extracts were dried $\left(\mathrm{MgSO}_{4}\right)$ and concentrated to give 2,2diphenyl-4-pentenylamine (S2) (15.2 g, 78\%) as a pale yellow, viscous oil.

A solution of $\mathbf{S 2}(3.01 \mathrm{~g}, 12.7 \mathrm{mmol})$ and benzaldehyde $(1.41 \mathrm{~g}, 13.3 \mathrm{mmol})$ in $\mathrm{MeOH}(50 \mathrm{~mL})$ was stirred at room temperature for $3.5 \mathrm{~h}$, treated with $\mathrm{NaBH}_{4}(720 \mathrm{mg}$, $19 \mathrm{mmol})$ and stirred overnight. The resulting mixture was treated with water $(100 \mathrm{~mL})$ and $1 \mathrm{M} \mathrm{NaOH}(30 \mathrm{~mL})$ and then extracted with $\mathrm{CH}_{2} \mathrm{Cl}_{2}(3 \times 100 \mathrm{~mL})$. The combined organic extracts were dried $\left(\mathrm{MgSO}_{4}\right)$ and concentrated. The resulting oily residue was chromatographed (hexanes-EtOAc $=8: 1)$ to give $\mathbf{1}(3.48 \mathrm{~g}, 84 \%)$ as a viscous oil that formed a white solid upon standing.

For S1: ${ }^{\mathrm{S} 2}{ }^{1} \mathrm{H}$ NMR: $\delta$ 7.30-7.41 (m, $\left.10 \mathrm{H}\right), 5.72(\mathrm{tdd}, J=7.6,10.1,17.1 \mathrm{~Hz}, 1$ $\mathrm{H}), 5.16-5.24(\mathrm{~m}, 2 \mathrm{H}), 3.13-3.16(\mathrm{~m}, 2 \mathrm{H}) . \quad{ }^{13} \mathrm{C}\left\{{ }^{1} \mathrm{H}\right\}$ NMR: $\delta$ 139.9, 131.9, 129.0, $128.1,127.2,122.1,120.5,51.8,44.0$.

For S2: ${ }^{\mathrm{S} 3}{ }^{1} \mathrm{H}$ NMR: $\delta$ 7.12-7.32 (m, $\left.10 \mathrm{H}\right), 5.41(\mathrm{tdd}, J=7.0,10.1,14.0 \mathrm{~Hz}, 1$ H), 4.97-5.09 (m, $2 \mathrm{H}), 3.34$ (s, $2 \mathrm{H}), 2.93-2.95(\mathrm{~m}, 2 \mathrm{H}), 0.84$ (br s, $2 \mathrm{H}) .{ }^{13} \mathrm{C}\left\{{ }^{1} \mathrm{H}\right\}$ NMR: $\delta 146.4,134.7,128.3,128.2,126.2,117.8,51.5,48.7,41.2$.

For 1: White solid. $\mathrm{mp} 44-45{ }^{\circ} \mathrm{C} . \quad \mathrm{TLC}$ (hexanes-EtOAc $=8: 1$ ): $\mathrm{R}_{f}=0.30 .{ }^{1} \mathrm{H}$ NMR: $\delta$ 7.09-7.28 (m, $15 \mathrm{H}), 5.33(\mathrm{tdd}, J=7.2,10.1,17.1 \mathrm{~Hz}, 1 \mathrm{H}), 4.87-5.00(\mathrm{~m}, 2 \mathrm{H})$, 
$3.71(\mathrm{~s}, 2 \mathrm{H}), 3.19$ (s, $2 \mathrm{H}), 3.02-3.04(\mathrm{~m}, 2 \mathrm{H}), 0.90$ (br s, $1 \mathrm{H}) .{ }^{13} \mathrm{C}\left\{{ }^{1} \mathrm{H}\right\}$ NMR: $\delta$ $146.9,140.8,135.0,128.3,128.2,128.1,126.8,126.1,117.7,55.4,54.3,50.3,41.7$. IR (neat, $\mathrm{cm}^{-1}$ ): 3026, 2819, 1494, 1447, 696. Anal. calcd (found) for $\mathrm{C}_{24} \mathrm{H}_{25} \mathrm{~N}: \mathrm{H}, 7.70$ (7.85); C, 88.03 (88.24); N, 4.28 (3.91).

The substituted arylmethyl(2,2-diphenyl-4-pentenyl)amines (4-bromobenzyl)(2,2-diphenyl-4-pentenyl)amine (S3, Table 1, entry 10), 4-[(2,2-diphenyl-4pentenylamino)-methyl]benzonitrile (S4, Table 1, entry 11), (2,2-diphenyl-4-pentenyl)(4-nitrobenzyl)amine (S5, Table 1, entry 12), methyl 4-[(2,2-diphenyl-4-pentenylamino)methyl]benzoate (S6, Table 1, entry 13), benzo[1,3]dioxol-5-ylmethyl-(2,2-diphenyl-4pentenyl)amine (S7, Table 1, entry 14) were synthesized via reductive amination of S2 with the appropriate aldehyde employing a procedure similar to that used to synthesize $\mathbf{1}$ (Scheme S1).

For S3: White solid. mp $54-56{ }^{\circ} \mathrm{C} . \quad$ TLC (hexanes-EtOAc $=5: 1$ ): $\mathrm{R}_{f}=0.48 .{ }^{1} \mathrm{H}$ NMR: $\delta$ 7.07-7.40 (m, $14 \mathrm{H}), 5.33(\mathrm{tdd}, J=7.2,10.1,17.1 \mathrm{~Hz}, 1 \mathrm{H}), 4.89-5.02(\mathrm{~m}, 2 \mathrm{H})$, 3.66 (s, $2 \mathrm{H}), 3.17$ (s, $2 \mathrm{H}), 3.03$ (d, $J=7.2 \mathrm{~Hz}, 2 \mathrm{H}), 0.87$ (br s, $1 \mathrm{H}) .{ }^{13} \mathrm{C}\left\{{ }^{1} \mathrm{H}\right\}$ NMR: $\delta$ 146.8, 139.7, 134.9, 131.4, 129.8, 128.1, 126.2, 120.6, 117.8, 55.3, 53.6, 50.2, 41.7. IR (neat, $\mathrm{cm}^{-1}$ ): $3059,2827,1488,1445,755,698$. Anal. calcd (found) for $\mathrm{C}_{24} \mathrm{H}_{24} \mathrm{BrN}: \mathrm{H}$, 5.95 (6.05); C, 70.94 (71.03); N, 3.45 (3.49).

For S4: Pale yellow solid. mp $74-75^{\circ} \mathrm{C} . \quad$ TLC (hexanes-EtOAc $=4: 1$ ): $\mathrm{R}_{f}=$ 0.40. ${ }^{1} \mathrm{H}$ NMR: $\delta 7.53(\mathrm{~d}, J=8.2 \mathrm{~Hz}, 2 \mathrm{H}), 7.29(\mathrm{~d}, J=8.2 \mathrm{~Hz}, 2 \mathrm{H}), 7.12-7.27(\mathrm{~m}, 10$ H), 5.29 (tdd, $J=7.0,10.1,17.1 \mathrm{~Hz}, 1 \mathrm{H}), 4.86-4.99$ (m, $2 \mathrm{H}), 3.74$ (s, $2 \mathrm{H}), 3.15$ (s, 2 H), $3.01(\mathrm{~d}, J=7.0 \mathrm{~Hz}, 2 \mathrm{H}), 0.95$ (br s, $1 \mathrm{H}) .{ }^{13} \mathrm{C}\left\{{ }^{1} \mathrm{H}\right\}$ NMR: $\delta$ 146.7, 146.5, 134.8, 132.2, 128.6, 128.2, 128.1, 126.3, 119.2, 117.8, 110.6, 55.4, 53.8, 50.2, 41.6. IR (neat, 
$\mathrm{cm}^{-1}$ ): 3023, 2851, 2226, 1450, 756, 698. Anal. calcd (found) for $\mathrm{C}_{25} \mathrm{H}_{24} \mathrm{~N}_{2}: \mathrm{H}, 6.68$ (6.96); C, 85.19 (84.93); N, 7.95 (8.08).

For S5: Pale yellow solid. $\mathrm{mp} 64-66{ }^{\circ} \mathrm{C}$. TLC (hexanes-EtOAc $\left.=4: 1\right): \mathrm{R}_{f}=$ 0.50. ${ }^{1} \mathrm{H}$ NMR: $\delta 8.09-8.11(\mathrm{~m}, 2 \mathrm{H}), 7.12-7.35(\mathrm{~m}, 12 \mathrm{H}), 5.25(\mathrm{tdd}, J=7.0,10.1,17.1$ Hz, 2 H), 3.78 (s, 2 H), 3.16 (s, $2 \mathrm{H}), 3.02(\mathrm{~d}, J=7.0 \mathrm{~Hz}, 2 \mathrm{H}), 0.96$ (br s, $1 \mathrm{H}) .{ }^{13} \mathrm{C}\left\{{ }^{1} \mathrm{H}\right\}$ NMR: $\delta 148.5,147.1,146.6,134.8,128.6,128.2,128.1,129.3,123.6,117.9,55.5,53.5$, 50.2, 41.7. IR (neat, $\mathrm{cm}^{-1}$ ): 3350, 3057, 2802, 1519, 1340, 696. Anal. calcd (found) for $\mathrm{C}_{24} \mathrm{H}_{24} \mathrm{~N}_{2} \mathrm{O}_{2}: \mathrm{H}, 6.49$ (6.60); C, $77.39(77.45) ; \mathrm{N}, 7.52$ (7.66).

For S6: White solid, 86\%. mp 71-72 ${ }^{\circ} \mathrm{C} .{ }^{1} \mathrm{H}$ NMR: $\delta 7.92(\mathrm{~d}, J=8.0 \mathrm{~Hz}, 2 \mathrm{H})$, 7.12-7.26 (m, $12 \mathrm{H}), 5.30$ (tdd, $J=7.2,9.7,17.1 \mathrm{~Hz}, 1 \mathrm{H}), 4.86-4.99$ (m, $2 \mathrm{H}), 3.88$ (s, 3 H), 3.74 (s, $2 \mathrm{H}), 3.15(\mathrm{~s}, 2 \mathrm{H}), 3.02(\mathrm{~d}, J=7.0 \mathrm{~Hz}, 2 \mathrm{H}), 0.85(\mathrm{br} \mathrm{s}, 1 \mathrm{H}) .{ }^{13} \mathrm{C}\left\{{ }^{1} \mathrm{H}\right\}$ NMR: $\delta 167.2,146.8,146.3,134.9,129.7,128.8,128.1,127.9,126.2,117.8,55.4,53.9$, 52.2, 52.1, 50.3, 41.7. IR (neat, $\mathrm{cm}^{-1}$ ): 2832, 1711, 1441, 1275, 1108, 695. Anal. calcd (found) for $\mathrm{C}_{26} \mathrm{H}_{27} \mathrm{NO}_{2}$ : H, 7.06 (7.09); C, 81.01 (80.97); N, 3.63 (3.64).

For S7: Viscous colorless oil. TLC (hexanes-EtOAc $=4: 1$ ): $\mathrm{R}_{f}=0.50 .{ }^{1} \mathrm{H}$ NMR: $\delta$ 7.12-7.25 (m, $10 \mathrm{H}), 6.61-6.70(\mathrm{~m}, 3 \mathrm{H}), 5.90$ (s, $2 \mathrm{H}), 5.31$ (tdd, $J=7.3,9.9$, $17.3 \mathrm{~Hz}, 1 \mathrm{H}), 4.87-5.00(\mathrm{~m}, 2 \mathrm{H}), 3.59$ (s, $2 \mathrm{H}), 3.16$ (s, $2 \mathrm{H}), 3.01$ (d, $J=7.0 \mathrm{~Hz}, 2 \mathrm{H})$, 0.82 (br s, $1 \mathrm{H}) .{ }^{13} \mathrm{C}\left\{{ }^{1} \mathrm{H}\right\}$ NMR: $\delta \quad 147.7,146.9,146.5,134.9,134.6,128.2,128.1$, 126.1, 121.1, 117.8, 108.7, 108.0, 100.9, 55.1, 54.0, 50.2, 41.7. IR (neat, $\mathrm{cm}^{-1}$ ): 2898, 1490, 1443, 1244, 1038, 699. Anal. calcd (found) for $\mathrm{C}_{25} \mathrm{H}_{25} \mathrm{NO}_{2}: \mathrm{H}, 6.78$ (6.86); C, 80.83 (80.85); N, 3.77 (3.92).

Benzyl(2,2-diphenyl-4-pentenyl)amine- ${ }^{15} \mathrm{~N}\left(1-{ }^{15} \mathrm{~N}\right) . \quad 1-{ }^{15} \mathrm{~N}$ was synthesized in $4 \%$ overall yield in three steps from S1 (Scheme S2). 


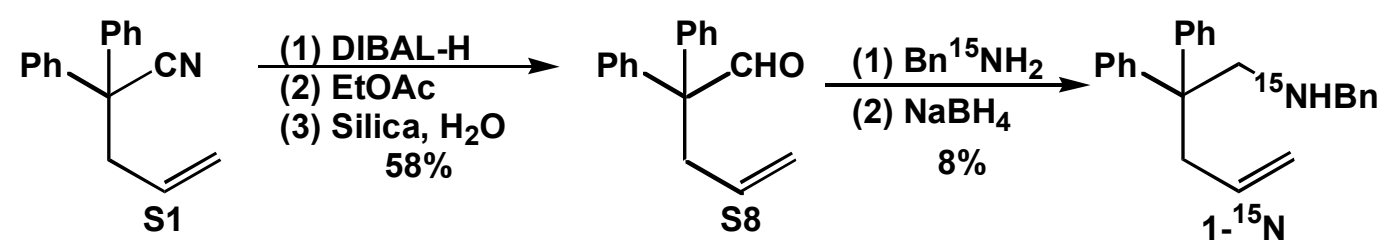

\section{Scheme S2}

DIBAL-H (4.6 mL, $26 \mathrm{mmol})$ was added over $10 \mathrm{~min}$ to a solution of $\mathbf{S 1}(1.50 \mathrm{~g}$, $6.43 \mathrm{mmol})$ in hexanes $(250 \mathrm{~mL})$ at $-78{ }^{\circ} \mathrm{C}$ and the resulting mixture was stirred for $1.5 \mathrm{~h}$ and quenched by slow addition of EtOAc $(16 \mathrm{~mL})$. The resulting suspension was stirred for 30 min at $-78{ }^{\circ} \mathrm{C}$, treated with an aqueous suspension of silica gel in water [5\% by weight, $25 \mathrm{~mL}$ ], allowed to stand for $30 \mathrm{~min}$, and then warmed slowly to room temperature. The resulting mixture was filtered through a plug of Celite, which was eluted with ether $(100 \mathrm{~mL})$. The resulting solution was washed with water $(100 \mathrm{~mL}), 1$ $\mathrm{M} \mathrm{HCl}(2 \times 100 \mathrm{~mL})$, saturated aqueous $\mathrm{NaHCO}_{3}(100 \mathrm{~mL})$, and brine $(100 \mathrm{~mL})$, dried $\left(\mathrm{MgSO}_{4}\right)$, and concentrated. The resulting oil was chromatographed (hexanes-EtOAc $=$ 8:1) to give 2,2-diphenyl-4-pentenal (S8) (0.88 g, 58\%).

A solution of ${ }^{15} \mathrm{~N}$-benzylamine (350 mg, $\left.3.24 \mathrm{mmol}\right)$ and $\mathbf{S 8}(740 \mathrm{mg}, 3.13 \mathrm{mmol})$ in $\mathrm{MeOH}(20 \mathrm{~mL})$ was stirred at room temperature for $20 \mathrm{~h}$, treated with $\mathrm{NaBH}_{4}(239 \mathrm{mg}$, $6.32 \mathrm{mmol})$, and stirred for an additional $4 \mathrm{~h}$. Water $(50 \mathrm{~mL})$ and $1 \mathrm{M} \mathrm{NaOH}(10 \mathrm{~mL})$ were added sequentially and the resulting mixture was extracted with $\mathrm{CH}_{2} \mathrm{Cl}_{2}(3 \times 50$ $\mathrm{mL}$ ). The combined organic extracts were washed with a 4:1 mixture of brine and $1 \mathrm{M}$ $\mathrm{NaOH}(2 \times 25 \mathrm{~mL})$, dried $\left(\mathrm{MgSO}_{4}\right)$, and concentrated. The resulting oily residue was 
chromatographed (hexanes-EtOAc $=8: 1)$ to give $1-{ }^{15} \mathrm{~N}(87 \mathrm{mg}, 8 \%)$ as a viscous oil that solidified upon standing.

For S8: ${ }^{\mathrm{S} 3}{ }^{1} \mathrm{H}$ NMR: $\delta \quad 5.81(\mathrm{tdd}, J=7.0,10.1,17.1 \mathrm{~Hz}, 1 \mathrm{H}), 5.15-5.22(\mathrm{~m}, 2$ H), 2.45-2.50 (m, $1 \mathrm{H}), 2.27-2.42(\mathrm{~m}, 2 \mathrm{H}), 1.82-1.94(\mathrm{~m}, 1 \mathrm{H}), 1.07$ (d, J = 6.8 Hz, $6 \mathrm{H})$. ${ }^{13} \mathrm{C}\left\{{ }^{1} \mathrm{H}\right\}$ NMR: $\delta$ 133.7, 120.8, 118.6, 39.2, 34.4, 29.6, 21.1, 18.7. IR (neat, $\mathrm{cm}^{-1}$ ): 3081, 2967, 2237, 1643, 1465, 922. TLC (hexanes-EtOAc $=8: 1): \mathrm{R}_{f}=0.41$. HRMS calcd (found) for $\mathrm{C}_{8} \mathrm{H}_{13} \mathrm{~N}\left(\mathrm{M}^{+}\right): 123.1048$ (123.1053)

For $1-{ }^{15} \mathbf{N}:{ }^{1} \mathrm{H}$ NMR: $\delta$ 7.21-7.34 (m, $\left.15 \mathrm{H}\right), 5.40(\mathrm{tdd}, J=7.0,10.1,17.1 \mathrm{~Hz}, 1$ H), 4.94-5.07 (m, 2 H), 3.77 (s, 2 H), 3.26 (s, 2 H), 3.10 (d, J = 7.2 Hz, 2 H), 1.00 (br s, 1 H). ${ }^{13} \mathrm{C}\left\{{ }^{1} \mathrm{H}\right\} \mathrm{NMR}: \delta 146.9,140.8,135.0,128.3,128.2,128.1,126.8,126.1,117.7$, $55.4(\mathrm{~d}, J=4.6 \mathrm{~Hz}), 54.3(\mathrm{~m}), 50.2,41.7$.

Benzyl(2,2-dimethyl-4-pentenyl)amine (S11, Table 1, entry 1). S11 was synthesized in three steps in $40 \%$ overall yield from isobutyronitrile as outlined in Scheme S3.

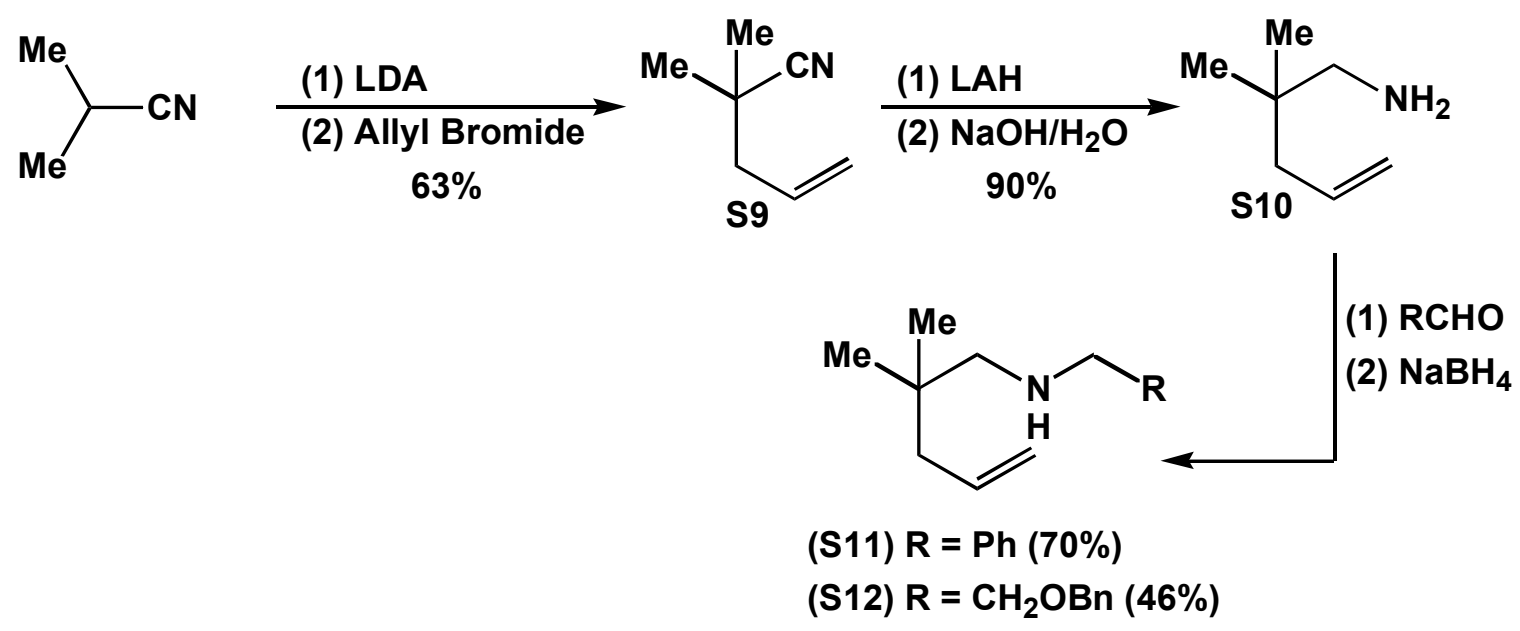

Scheme S3 
Isobutyronitrile ( $8.49 \mathrm{~g}, 123 \mathrm{mmol})$ was added to a solution of LDA [generated in situ from $n$-BuLi (52 mL in hexanes, $2.5 \mathrm{M}, 130 \mathrm{mmol})$ and diisopropylamine $(12.4 \mathrm{~g}$, $123 \mathrm{mmol})$ in THF $(300 \mathrm{~mL})]$ at $-78^{\circ} \mathrm{C}$ and stirred for $45 \mathrm{~min}$. To the resulting solution was added allyl bromide $(21.5 \mathrm{~mL}, 248 \mathrm{mmol})$. The solution was warmed to room temperature overnight with stirring. $\mathrm{CH}_{2} \mathrm{Cl}_{2}(75 \mathrm{~mL})$ was added and the resulting biphasic mixture washed with water $(3 \times 150 \mathrm{~mL})$, dried $\left(\mathrm{MgSO}_{4}\right)$, and concentrated. The residue was distilled under reduced pressure to give 2,2-dimethyl-4-pentenenitrile (S9) $(8.43 \mathrm{~g}, 63 \%)$.

Conversion of S9 to $\mathbf{S 1 1}$ was accomplished in a manner similar to that employed for the conversion of $\mathbf{S 1}$ to $\mathbf{1}$.

For S9: ${ }^{\mathrm{S} 1} \quad{ }^{1} \mathrm{H}$ NMR: $\delta 5.86(\mathrm{tdd}, J=7.3,10.3,16.9 \mathrm{~Hz}, 1 \mathrm{H}), 5.15-5.24(\mathrm{~m}, 2$ H), 2.27 (m, $2 \mathrm{H}), 1.34(\mathrm{~s}, 6 \mathrm{H}) .{ }^{13} \mathrm{C}\left\{{ }^{1} \mathrm{H}\right\}$ NMR: $\delta$ 132.3, 124.9, 120.0, 45.2, 32.3, 26.3.

For 2,2-dimethyl-4-pentenylamine (S10): ${ }^{\mathrm{S}}$ Colorless solution in ether. ${ }^{1} \mathrm{H}$ NMR: $\delta \quad 5.74-5.84(\mathrm{~m}, 1 \mathrm{H}), 4.98-5.03(\mathrm{~m}, 2 \mathrm{H}), 2.44(\mathrm{~s}, 2 \mathrm{H}), 1.95(\mathrm{td}, J=0.9,7.5 \mathrm{~Hz}$, $2 \mathrm{H}), 1.18(\mathrm{~s}, 6 \mathrm{H}) .{ }^{13} \mathrm{C}\left\{{ }^{1} \mathrm{H}\right\}$ NMR: $\delta 135.3,117.1,52.7,44.1,34.9,24.7$.

For S11: ${ }^{\mathrm{S} 5}$ TLC (hexanes-EtOAc $\left.=4: 1\right): \mathrm{R}_{f}=0.29 .{ }^{1} \mathrm{H}$ NMR: $\delta$ 7.23-7.37 $(\mathrm{m}$, 5 H), 5.74-5.84 (m, $1 \mathrm{H}), 4.98-5.03(\mathrm{~m}, 2 \mathrm{H}), 2.38(\mathrm{~s}, 2 \mathrm{H}), 2.03$ (m, $2 \mathrm{H}), 0.90(\mathrm{~s}, 6 \mathrm{H})$. ${ }^{13} \mathrm{C}\left\{{ }^{1} \mathrm{H}\right\}$ NMR: $\delta 135.6,128.5,128.2,127.0,117.0,59.5,54.7,44.8,34.4,25.6$. IR (neat, $\mathrm{cm}^{-1}$ ): 2955, 2360, 1457, 912, 734, 697. Anal. calcd (found) for $\mathrm{C}_{14} \mathrm{H}_{21} \mathrm{~N}: \mathrm{H}$, 10.41 (10.25); C, $82.70(82.59) ; \mathrm{N}, 6.89$ (6.83).

(2-Benzyloxyethyl)(2,2-dimethyl-4-pentenyl)amine (S12, Table 1, entry 2). Compound S12 was synthesized from isoburyronitrile in $46 \%$ yield employing a procedure similar to that used to synthesize S11 (Scheme S3). TLC (hexanes-EtOAc $=$ 
3:2): $\mathrm{R}_{f}=0.30 .{ }^{1} \mathrm{H}$ NMR (Figure $\left.\mathrm{S} 1\right): \delta$ 7.26-7.39 (m, $\left.5 \mathrm{H}\right)$, 5.76-5.87 (m, $\left.1 \mathrm{H}\right)$, 4.98$5.03(\mathrm{~m}, 2 \mathrm{H}), 4.54(\mathrm{~s}, 2 \mathrm{H}), 3.60(\mathrm{t}, J=5.0 \mathrm{~Hz}, 2 \mathrm{H}), 2.81(\mathrm{t}, J=5.1 \mathrm{~Hz}, 2 \mathrm{H}), 2.36(\mathrm{~s}, 2$ H), 2.00-2.02 (m, $2 \mathrm{H}), 1.32($ br s, $1 \mathrm{H}), 0.89(\mathrm{~s}, 6 \mathrm{H}) .{ }^{13} \mathrm{C}\left\{{ }^{1} \mathrm{H}\right\}$ NMR (Figure S2): $\delta$ $138.6,135.7,128.5,127.8,127.7,116.9,73.2,69.6,60.4,50.5,44.9,34.5,25.6$. IR (neat, $\left.\mathrm{cm}^{-1}\right): 2952,2865,1458,1095,736,697$. HRMS calcd (found) for $\mathrm{C}_{16} \mathrm{H}_{24} \mathrm{NO}\left(\mathrm{M}^{+}-\mathrm{H}\right)$ : $246.1858(246.1860)$.

Benzyl-2,2-dimethyl-4-pentenylamine (S13). S13 was synthesized from 2,2dimethyl-4-pentenylamine (S14) in 37\% yield employing a procedure similar to that used to convert $\mathbf{S 1}$ to $\mathbf{1}$.

For S14: ${ }^{\mathrm{S} 6}{ }^{1} \mathrm{H}$ NMR: $\delta 5.80(\operatorname{tdd}, J=6.5,10.3,17.1 \mathrm{~Hz}, 1 \mathrm{H}), 4.92-5.04(\mathrm{~m}, 2$ H), 2.70 (t, $J=7.1 \mathrm{~Hz}, 2 \mathrm{H}), 2.06-2.11(\mathrm{~m}, 2 \mathrm{H}), 1.50-1.57$ (m, $2 \mathrm{H}), 1.41$ (br. s, $2 \mathrm{H})$. ${ }^{13} \mathrm{C}\left\{{ }^{1} \mathrm{H}\right\}$ NMR: $\delta 138.5,114.8,41.8,32.8,31.2$.

For S13: ${ }^{\mathrm{S7}}$ TLC (hexanes-EtOAc $\left.=3: 2\right): \mathrm{R}_{f}=0.42 .{ }^{1} \mathrm{H}$ NMR: $\delta$ 7.23-7.33 $(\mathrm{m}$, $5 \mathrm{H}), 5.82$ (tdd, $J=6.7,10.3,16.9 \mathrm{~Hz}, 1 \mathrm{H}), 4.93-5.04(\mathrm{~m}, 2 \mathrm{H}), 3.80(\mathrm{~s}, 2 \mathrm{H}), 2.65(\mathrm{t}, J$ $=7.2 \mathrm{~Hz}, 2 \mathrm{H}), 2.13-2.08(\mathrm{~m}, 2 \mathrm{H}), 1.53-1.66(\mathrm{~m}, 3 \mathrm{H}) .{ }^{13} \mathrm{C}\left\{{ }^{1} \mathrm{H}\right\} \mathrm{NMR}: \delta$ 140.5, 138.6, $128.5,128.3,127.1,114.8,54.1,49.0,31.7,29.3$.

Cyclohexyl(2,2-dimethyl-4-pentenyl)amine (S15, Table 1, entry 3). A solution of cyclohexylamine $(1.72 \mathrm{~mL}, 14.8 \mathrm{mmol})$ and 2,2-dimethyl-4-pentenal (1.68 g, 15.0 mmol) in $\mathrm{MeOH}(50 \mathrm{~mL})$ was stirred at room temperature for $3 \mathrm{~h}$, treated with $\mathrm{NaBH}_{4}$ (1.13 g, $29.9 \mathrm{mmol})$, and stirred for an additional $19 \mathrm{~h}$. Water $(100 \mathrm{~mL})$ and $1 \mathrm{M} \mathrm{NaOH}$ (30 mL) were added sequentially and the resulting mixture was extracted with $\mathrm{CH}_{2} \mathrm{Cl}_{2}$ (3 $\times 100 \mathrm{~mL})$. The combined organic extracts were dried $\left(\mathrm{MgSO}_{4}\right)$ and concentrated. The resulting oily residue was chromatographed (hexanes-EtOAc $=3: 1)$ to give $\mathbf{S 1 5}(1.53 \mathrm{~g}$, 
52\%). TLC (hexanes-EtOAc $=3: 1): \quad \mathrm{R}_{f}=0.60 .{ }^{1} \mathrm{H}$ NMR: $\delta \quad 5.76-5.86(\mathrm{~m}, 1 \mathrm{H}), 4.97-$ $5.03(\mathrm{~m}, 2 \mathrm{H}), 2.36(\mathrm{~s}, 2 \mathrm{H}), 2.27-2.34(\mathrm{~m}, 1 \mathrm{H}), 1.99(\mathrm{~d}, J=7.5 \mathrm{~Hz}, 2 \mathrm{H}), 1.82-1.86(\mathrm{~m}$, $2 \mathrm{H}), 1.68-1.73$ (m, $2 \mathrm{H}), 1.56-1.61$ (m, $1 \mathrm{H}), 1.12-1.29$ (m, $4 \mathrm{H}), 1.08-1.10(\mathrm{~m}, 2 \mathrm{H})$, 0.87 (s, $6 \mathrm{H}) .{ }^{13} \mathrm{C}\left\{{ }^{1} \mathrm{H}\right\}$ NMR: $\delta \quad 135.8,116.7,57.8,57.6,44.8,34.2,33.9,26.4,25.6$, 25.3. IR (neat, $\mathrm{cm}^{-1}$ ): 2926, 2854, 1452, 1124, 911. Anal. calcd (found) for $\mathrm{C}_{13} \mathrm{H}_{25} \mathrm{~N}$ : H, 12.90 (12.99); C, 79.93 (79.51); N, 7.17 (7.50).

(1-Allylcyclohexylmethyl)benzylamine (S22, Table 1, entry 4), benzyl[1-(2methylallyl)-cyclohexylmethyl]amine (S23, Table 1, entry 6), and benzyl[1-(3butenyl)cyclohexylmethyl]amine (S24, Table 1, entry 15) were synthesized employing a procedure similar to that used to synthesize S11 (Scheme S4).

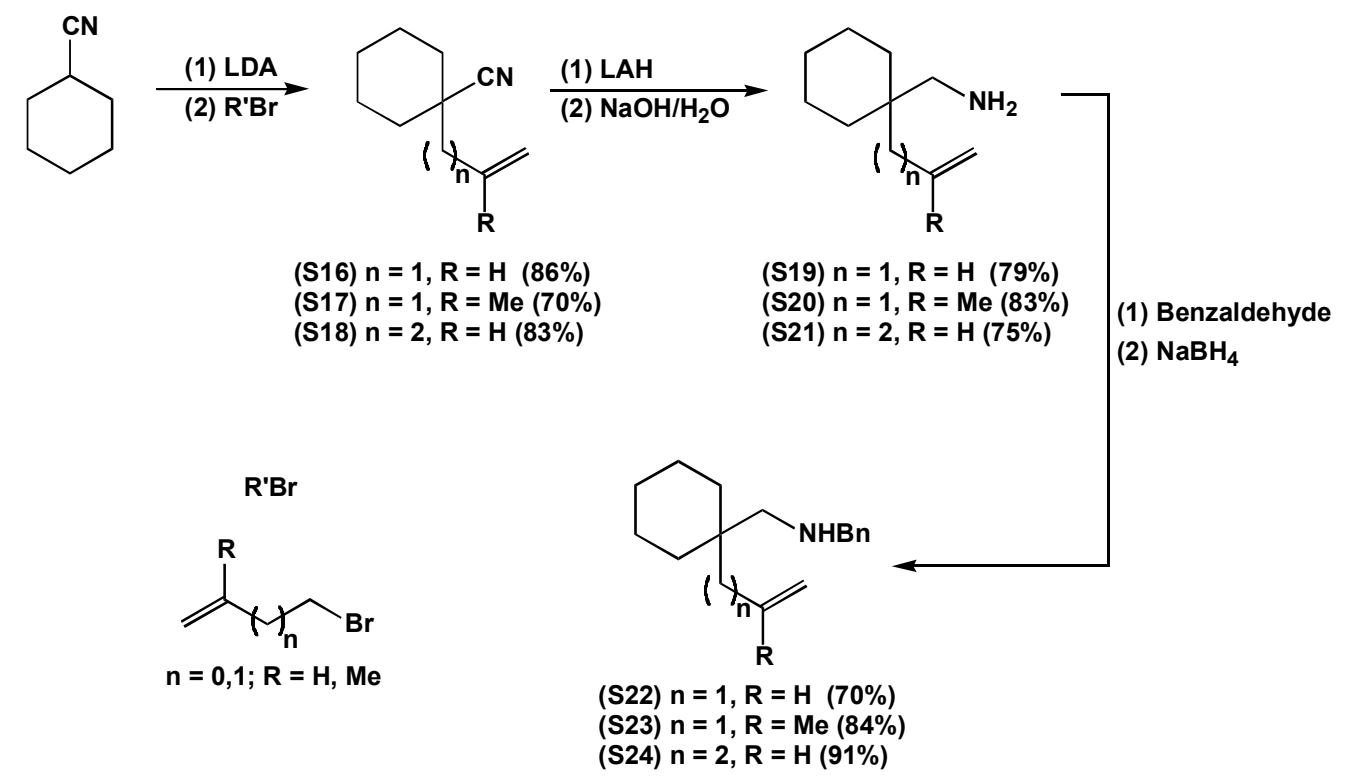

\section{Scheme S4}

For 1-allyl-cyclohexanecarbonitrile (S16): $\operatorname{TLC}($ hexanes-EtOAc $=12: 1): \mathrm{R}_{f}=$ 0.36. ${ }^{1} \mathrm{H}$ NMR: $\delta 5.88(\operatorname{tdd}, J=7.3,10.3,16.7 \mathrm{~Hz}, 1 \mathrm{H}), 5.13-5.21(\mathrm{~m}, 2 \mathrm{H}), 2.27(\mathrm{~d}, J$ 
$=7.3 \mathrm{~Hz}, 2 \mathrm{H}), 1.94(\mathrm{~d}, J=13.3 \mathrm{~Hz}, 2 \mathrm{H}), 1.55-1.73(\mathrm{~m}, 5 \mathrm{H}), 1.11-1.27(\mathrm{~m}, 3 \mathrm{H})$. ${ }^{13} \mathrm{C}\left\{{ }^{1} \mathrm{H}\right\}$ NMR: $\delta \quad 132.0,123.4,119.7,44.7,38.9,35.4,25.4,23.0$. IR (neat, $\mathrm{cm}^{-1}$ ): 3079, 2859, 2230, 1449, 922, 714. Anal. calcd (found) for $\mathrm{C}_{10} \mathrm{H}_{15} \mathrm{~N}$ : H, 10.13 (9.96); C, 80.48 (80.24); N, 9.39 (9.35).

For (1-allylcyclohexyl)methylamine (S19): ${ }^{\mathrm{S} 8} \quad{ }^{1} \mathrm{H}$ NMR: $\delta \quad 5.79$ (tdd, $J=7.5$, 10.6, $16.4 \mathrm{~Hz}, 1 \mathrm{H}), 5.02-5.06$ (m, $2 \mathrm{H}), 2.51(\mathrm{~s}, 2 \mathrm{H}), 2.06$ (d, $J=7.5 \mathrm{~Hz}, 2 \mathrm{H}), 1.22-$ $1.43(\mathrm{~m}, 10 \mathrm{H}), 0.95$ (br s, $2 \mathrm{H}) .{ }^{13} \mathrm{C}\left\{{ }^{1} \mathrm{H}\right\}$ NMR: $\delta$ 135.2, 116.9, 49.0, 40.0, 37.2, 33.4, 26.6, 21.6.

For S22: ${ }^{55}$ TLC (hexanes-EtOAc $\left.=6: 1\right): \mathrm{R}_{f}=0.40 .{ }^{1} \mathrm{H}$ NMR: $\delta$ 7.20-7.33 (m, 5 H), 5.77 (tdd, $J=7.5,10.1,16.9 \mathrm{~Hz}, 1 \mathrm{H}), 4.97-5.03(\mathrm{~m}, 2 \mathrm{H}), 3.76(\mathrm{~s}, 2 \mathrm{H}), 2.41$ (s, 2 $\mathrm{H}), 2.10-2.12(\mathrm{~m}, 2 \mathrm{H}), 1.30-1.40(\mathrm{~m}, 10 \mathrm{H}), 1.11(\mathrm{br} \mathrm{s}, 1 \mathrm{H}) .{ }^{13} \mathrm{C}\left\{{ }^{1} \mathrm{H}\right\}$ NMR: $\delta$ 141.2, 135.5, 128.4, 128.1, 126.8, 116.7, 56.0, 54.9, 40.8, 36.8, 34.1, 26.6, 21.7. IR (neat, $\mathrm{cm}^{-1}$ ): 3067, 2922, 2851, 1451, 910, 733. Anal. calcd (found) for $\mathrm{C}_{17} \mathrm{H}_{25} \mathrm{~N}$ : H, 10.35 (10.33); C, 83.89 (83.96); N, 5.75 (5.67).

For 1-(2-methyl-2-propenyl)-cyclohexanecarbonitrile S17: ${ }^{\mathrm{S} 9} \quad{ }^{1} \mathrm{H}$ NMR: $\delta$ 4.94-4.96 (m, 1 H), 4.81-4.83 (m, 1 H), 2.25 (s, 2 H), 1.95-1.98 (m, 2 H), 1.89-1.90 (m, 3 $\mathrm{H}), 1.57-1.75$ (m, $5 \mathrm{H}), 1.10-1.26(\mathrm{~m}, 3 \mathrm{H}) .{ }^{13} \mathrm{C}\left\{{ }^{1} \mathrm{H}\right\}$ NMR: $\delta$ 140.3, 124.1, 116.1, 48.4, $38.0,36.1,25.4,24.1,23.1$.

For [1-(2-methyl-2-propenyl)-cyclohexyl]methylamine (S20): ${ }^{9}{ }^{1} \mathrm{H}$ NMR: $\delta$ 4.85-4.86 (m, 1 H), 4.66-4.67 (m, 1 H), 2.57 (s, 2 H), 2.02 (s, 2 H), 1.79-1.80 (m, 3 H), 1.35-1.51 (m, $6 \mathrm{H}), 1.25-1.32(\mathrm{~m}, 4 \mathrm{H}), 0.99$ (br s, $2 \mathrm{H}) .{ }^{13} \mathrm{C}\left\{{ }^{1} \mathrm{H}\right\}$ NMR: $\delta$ 143.6, 114.4, $48.4,43.9,38.0,34.0,26.5,25.6,21.9$. 
For S23: ${ }^{\mathrm{S} 5}$ TLC (hexanes-EtOAc $\left.=5: 1\right): \mathrm{R}_{f}=0.43 .{ }^{1} \mathrm{H}$ NMR: $\delta$ 7.22-7.35 $(\mathrm{m}$, 5 H), 4.84-4.87 (m, 1 H), 4.66-4.67 (m, 1 H), 3.76 (s, 2 H), 2.48 (s, 2 H), $2.11(\mathrm{~s}, 2 \mathrm{H})$, $1.78(\mathrm{~s}, 3 \mathrm{H}), 1.30-1.46(\mathrm{~m}, 10 \mathrm{H}), 1.11(\mathrm{br} \mathrm{s}, 1 \mathrm{H}) .{ }^{13} \mathrm{C}\left\{{ }^{1} \mathrm{H}\right\}$ NMR: $\delta$ 143.8, 141.2, $128.4,128.2,126.8,114.1,55.6,54.9,44.4,37.5,34.7,26.5,25.6,21.9$. IR (neat, $\mathrm{cm}^{-1}$ ): 2924, 2853, 1640, 1452, 734, 697. Anal. calcd (found) for $\mathrm{C}_{18} \mathrm{H}_{27} \mathrm{~N}: \mathrm{H}, 10.57$ (10.70); C, 83.99 (83.97); N, 5.44 (5.67).

For 1-(3-butenyl)cyclohexanecarbonitrile (S18): TLC (hexanes-EtOAc $=5: 1$ ): $\mathrm{R}_{f}=0.52 .{ }^{1} \mathrm{H}$ NMR: $\delta 5.81(\mathrm{tdd}, J=6.7,10.3,16.9 \mathrm{~Hz}, 1 \mathrm{H}), 4.97-5.09(\mathrm{~m}, 2 \mathrm{H}), 2.22-$ $2.27(\mathrm{~m}, 2 \mathrm{H}), 1.98(\mathrm{~d}, J=12.5 \mathrm{~Hz}, 2 \mathrm{H}), 1.55-1.75(\mathrm{~m}, 7 \mathrm{H}), 1.11-1.26(\mathrm{~m}, 3 \mathrm{H})$. ${ }^{13} \mathrm{C}\left\{{ }^{1} \mathrm{H}\right\}$ NMR: $\delta 137.4,123.6,115.4,39.8,38.9,35.8,28.8,25.5,23.2$. IR (neat, $\mathrm{cm}^{-1}$ ): 2934, 2859, 2230, 1643, 1451, 914. Anal. calcd (found) for $\mathrm{C}_{11} \mathrm{H}_{17} \mathrm{~N}: \mathrm{H}, 10.50$ (10.65); C, $80.93(80.85) ; \mathrm{N}, 8.58$ (8.79).

For 1-(3-butenyl-cyclohexyl)methylamine (S21): $\mathrm{TLC}\left(\mathrm{CH}_{2} \mathrm{Cl}_{2}-\mathrm{MeOH}=9: 1\right)$ : $\mathrm{R}_{f}=0.50 .{ }^{1} \mathrm{H}$ NMR: $\delta 5.81(\mathrm{tdd}, J=6.5 \mathrm{~Hz}, 10.3,16.9 \mathrm{~Hz}, 1 \mathrm{H}), 4.87-5.02(\mathrm{~m}, 2 \mathrm{H})$, 2.50 (s, $2 \mathrm{H}), 1.89-1.95(\mathrm{~m}, 2 \mathrm{H}), 1.18-1.44(\mathrm{~m}, 12 \mathrm{H}), 0.91$ (br s, $2 \mathrm{H}) .{ }^{13} \mathrm{C}\left\{{ }^{1} \mathrm{H}\right\}$ NMR: $\delta$ 139.7, 114.0, 48.5, 36.4, 34.2, 33.5, 27.5, 26.6, 21.6. IR (neat, $\mathrm{cm}^{-1}$ ): 2922, 2853, 1640, 1454, 907, 800. Anal. calcd (found) for $\mathrm{C}_{11} \mathrm{H}_{21} \mathrm{~N}$ : H, 12.65 (12.60); C, 78.97 (78.76); N, $8.37(8.23)$.

For S24: $\mathrm{TLC}($ hexanes-EtOAc $=5: 1): \mathrm{R}_{f}=0.46 .{ }^{1} \mathrm{H}$ NMR: $\delta$ 7.23-7.35 $(\mathrm{m}, 5$ H), 5.83 (tdd, $J=6.5,10.3,17.1 \mathrm{~Hz}, 1 \mathrm{H}), 4.91-5.02(\mathrm{~m}, 2 \mathrm{H}), 3.79$ (s, $2 \mathrm{H}), 2.42$ (s, 2 H), 1.87-1.94 (m, $2 \mathrm{H}), 1.26-1.44(\mathrm{~m}, 12 \mathrm{H}), 1.09$ (br s, $1 \mathrm{H}) .{ }^{13} \mathrm{C}\left\{{ }^{1} \mathrm{H}\right\}$ NMR: $\delta$ 141.2, $140.0,128.4,128.1,126.9,113.9,55.6,54.8,36.1,35.3,34.4,27.7,26.6,21.7$. IR (neat, 
$\mathrm{cm}^{-1}$ ): 2923, 2852, 1451, 907, 734, 697. Anal. calcd (found) for $\mathrm{C}_{18} \mathrm{H}_{27} \mathrm{~N}: \mathrm{H}, 10.57$ (10.39); C, 83.99 (83.86); N, 5.44 (5.32).

Benzyl(2-isopropyl-4-pentenyl)amine (S25, Table 1, entry 8). Compound S25 was synthesized in $25 \%$ overall yield in three steps from isovaleronitrile employing a procedure similar to that used to synthesize S11.

For 2-isopropyl-4-pentenenitrile (S26): TLC (hexanes-EtOAc $=8: 1): \quad \mathrm{R}_{f}=$ 0.41. ${ }^{1} \mathrm{H}$ NMR: $\delta 5.81(\mathrm{tdd}, J=7.0,10.1,17.1 \mathrm{~Hz}, 1 \mathrm{H}), 5.15-5.22(\mathrm{~m}, 2 \mathrm{H}), 2.45-2.50$ (m, $1 \mathrm{H}), 2.27-2.42(\mathrm{~m}, 2 \mathrm{H}), 1.82-1.94(\mathrm{~m}, 1 \mathrm{H}), 1.07(\mathrm{~d}, J=6.8 \mathrm{~Hz}, 6 \mathrm{H}) .{ }^{13} \mathrm{C}\left\{{ }^{1} \mathrm{H}\right\}$ NMR: $\delta 133.7,120.8,118.6,39.2,34.4,29.6,21.1,18.7$. IR (neat, $\left.\mathrm{cm}^{-1}\right): 3081,2967$, 2237, 1643, 1465, 922. HRMS: calcd (found) for $\mathrm{C}_{8} \mathrm{H}_{13} \mathrm{~N}\left(\mathrm{M}^{+}\right)$: 123.1048 (123.1053).

For S25: $57 \%$. TLC (hexanes-EtOAc $=2: 1$ ): $\mathrm{R}_{f}=0.36 .{ }^{1} \mathrm{H}$ NMR (Figure S3): $\delta$ 7.20-7.32 (m, 5 H), 5.78 (tdd, $J=7.2,9.9,17.1 \mathrm{~Hz}, 1 \mathrm{H}), 4.94-5.03(\mathrm{~m}, 2 \mathrm{H}), 3.75(\mathrm{~s}, 2$ H), 2.48-2.60 (m, 2 H), 2.09-2.16 (m, 1 H), 1.98-2.05 (m, 1 H), 1.73-1.85 (m, 1 H), 1.41$1.48(\mathrm{~m}, 1 \mathrm{H}), 1.30($ br s, $1 \mathrm{H}), 0.86(\mathrm{~d}, J=6.8 \mathrm{~Hz}, 3 \mathrm{H}), 0.85(\mathrm{~d}, J=6.8 \mathrm{~Hz}, 3 \mathrm{H})$. ${ }^{13} \mathrm{C}\left\{{ }^{1} \mathrm{H}\right\}$ NMR (Figure S4): $\delta 140.9,138.4,128.4,128.2,126.9,115.6,54.4,50.5,44.2$, 34.1, 28.6, 19.7, 19.2. IR (neat, $\mathrm{cm}^{-1}$ ): 3068, 2957, 1454, 909, 734, 697. HRMS calcd (found) for $\mathrm{C}_{15} \mathrm{H}_{23} \mathrm{~N}\left(\mathrm{M}^{+}\right)$: 217.1830 (217.1831).

Benzyl[2-(4-methoxyphenyl)-4-pentenyl]amine (S27, Table 1, entry 9). Compound S27 was synthesized in three steps in $20 \%$ overall yield from (4-methoxyphenyl)acetonitrile employing a procedure similar to that used to synthesize S11.

For 2-(4-methoxyphenyl)-4-pentenenitrile (S28): ${ }^{\mathrm{S} 2}{ }^{1} \mathrm{H}$ NMR: $\delta$ 7.23-7.25 (m, $2 \mathrm{H}), 6.88-6.92(\mathrm{~m}, 2 \mathrm{H}), 5.74-5.84(\mathrm{~m}, 1 \mathrm{H}), 5.19-5.21(\mathrm{~m}, 1 \mathrm{H}), 5.15-5.17(\mathrm{~m}, 1 \mathrm{H})$, 
3.78-3.82 (m, $4 \mathrm{H}), 2.54-2.67(\mathrm{~m}, 2 \mathrm{H}) .{ }^{13} \mathrm{C}\left\{{ }^{1} \mathrm{H}\right\}$ NMR: $\delta \quad 159.5,132.8,128.6,127.3$, $120.7,119.4,114.5,55.5,40.0,36.8$.

For 2-(4-methoxyphenyl)-4-pentenylamine (S29): $\quad \mathrm{TLC}\left(\mathrm{CH}_{2} \mathrm{Cl}_{2}-\mathrm{MeOH}=\right.$ 85:15): $\mathrm{R}_{f}=0.81 .{ }^{1} \mathrm{H}$ NMR: $\delta 7.09(\mathrm{~d}, J=8.7 \mathrm{~Hz}, 2 \mathrm{H}), 6.86(\mathrm{~d}, J=8.7 \mathrm{~Hz}, 2 \mathrm{H}), 5.67$ (tdd, $J=7.3,9.9,16.9 \mathrm{~Hz}, 1 \mathrm{H}), 4.90-5.00(\mathrm{~m}, 2 \mathrm{H}), 3.78$ (s, $3 \mathrm{H}), 2.93$ (dd, $J=5.1,12.6$ $\mathrm{Hz}, 1 \mathrm{H}), 2.79$ (dd, $J=8.7,12.6 \mathrm{~Hz}, 1 \mathrm{H}), 2.59-2.66(\mathrm{~m}, 1 \mathrm{H}), 2.27-2.41(\mathrm{~m}, 2 \mathrm{H}), 1.19$ (br s, $2 \mathrm{H}) .{ }^{13} \mathrm{C}\left\{{ }^{1} \mathrm{H}\right\}$ NMR: $\delta 159.3,136.8,135.1,128.9,116.1,114.0,55.3,48.6,47.7$, 38.6. IR (neat, $\mathrm{cm}^{-1}$ ): 2917, 1611, 1512, 1245, 1034, 826. Anal. calcd (found) for $\mathrm{C}_{12} \mathrm{H}_{17} \mathrm{NO}: 8.96$ (8.93); C, 75.35 (75.25); N, 7.32 (7.39).

For S27: $69 \% . \quad$ TLC (hexanes-EtOAc $=2: 1$ ): $\mathrm{R}_{f}=0.37 .{ }^{1} \mathrm{H}$ NMR: $\delta$ 7.21-7.32 (m, $5 \mathrm{H}), 7.11(\mathrm{~d}, J=8.5 \mathrm{~Hz}, 2 \mathrm{H}), 6.86(\mathrm{~d}, J=8.7 \mathrm{~Hz}, 2 \mathrm{H}), 5.68$ (tdd, $J=6.9,9.7,16.9$ Hz, $1 \mathrm{H}), 4.91-5.00(\mathrm{~m}, 2 \mathrm{H}), 3.80(\mathrm{~s}, 3 \mathrm{H}), 3.78(\mathrm{~d}, J=13.8 \mathrm{~Hz}, 1 \mathrm{H}), 3.70(\mathrm{~d}, J=13.4$ Hz, $1 \mathrm{H}), 2.84-2.91$ (m, 2 H), 2.73-2.79 (m, $1 \mathrm{H}), 2.28-2.43$ (m, $2 \mathrm{H}), 1.46$ (br s, $1 \mathrm{H})$. ${ }^{13} \mathrm{C}\left\{{ }^{1} \mathrm{H}\right\}$ NMR: $\delta \quad 158.3,140.4,136.8,135.3,128.8,128.4,128.1,127.0,116.1,114.1$, 55.3, 54.6, 53.9, 45.1, 39.3. IR (neat, $\mathrm{cm}^{-1}$ ): 2908, 1512, 1453, 1247, 1034, 828. Anal. calcd (found) for $\mathrm{C}_{19} \mathrm{H}_{23} \mathrm{NO}$ : H, 8.24 (8.32); C, 81.10 (81.00); N, 4.98 (5.36).

\section{Benzyl[1-(tert-butyldimethylsilyloxy-2-propenyl]cyclohexylamine (S33, Table}

1, entry 7). S33 was synthesized in four steps in $17 \%$ overall yield from cyclohexanecarbonitrile (Scheme S5). 


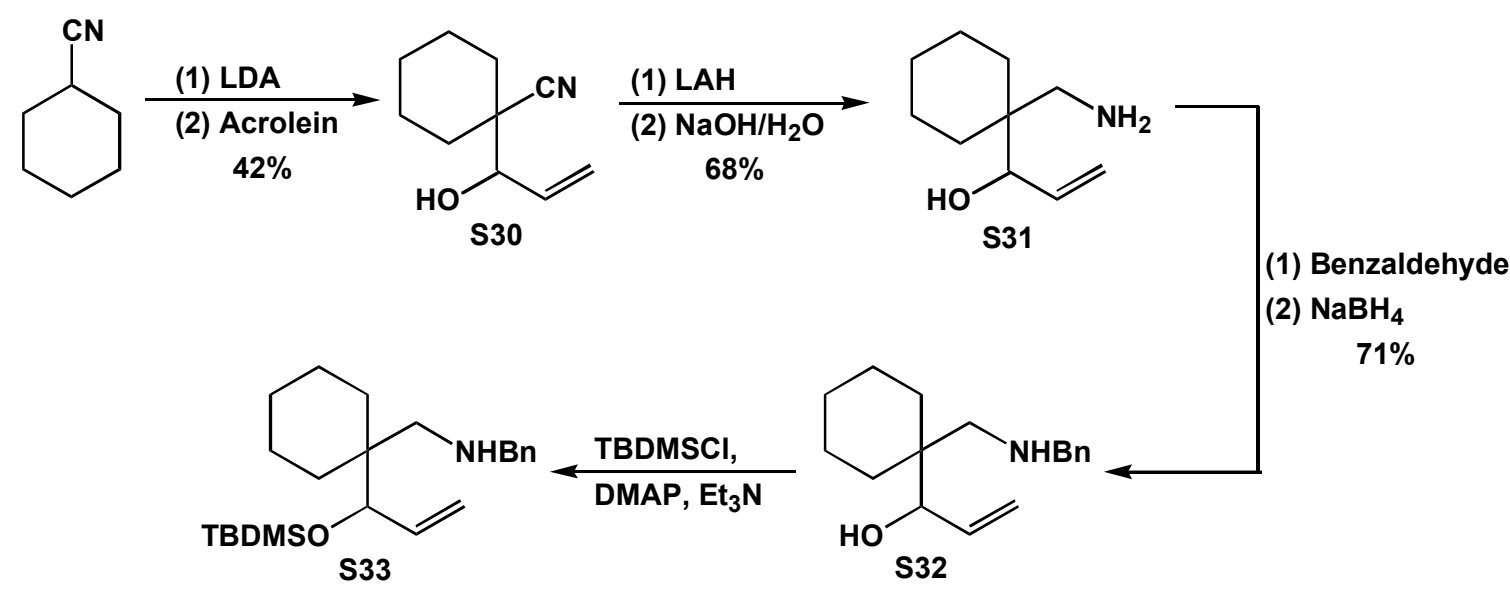

\section{Scheme S5}

Cyclohexanecarbonitrile $(7.80 \mathrm{~g}, 71.5 \mathrm{mmol})$ was added to a solution of LDA [generated from $n$-BuLi $(30 \mathrm{~mL}$ in hexanes, $2.5 \mathrm{M}, 75 \mathrm{mmol})$ and diisopropylamine $(7.22$ $\mathrm{g}, 71.5 \mathrm{mmol})$ in THF $(100 \mathrm{~mL})]$ at $-78{ }^{\circ} \mathrm{C}$ and stirred for $1 \mathrm{~h}$. Acrolein $(9.5 \mathrm{~mL}, 140$ mmol) was added via syringe and the resulting solution was stirred and warmed to room temperature overnight. Water $(100 \mathrm{~mL})$ was added, the layers were separated, and the aqueous layer was extracted with ether $(3 \times 75 \mathrm{~mL})$. The combined ether extracts were washed with water $(2 \times 100 \mathrm{~mL})$ and brine $(50 \mathrm{~mL})$, dried $\left(\mathrm{MgSO}_{4}\right)$, and concentrated. A solution of the resulting oily residue and benzylamine $(1.0 \mathrm{~mL}, 7.0 \mathrm{mmol})$ in $\mathrm{MeOH}(50$ mL) was stirred for $4 \mathrm{~h}$, treated with $\mathrm{NaBH}_{4}(0.5 \mathrm{~g}, 0.13 \mathrm{mmol})$ and stirred for an additional $4 \mathrm{~h}$. The resulting mixture was diluted with $\mathrm{CH}_{2} \mathrm{Cl}_{2}(100 \mathrm{~mL})$, washed with 1 $\mathrm{M} \mathrm{HCl}(2 \times 100 \mathrm{~mL})$ give 1-(1-hydroxy-2-propenyl)-cyclohexanecarbonitrile (S30) $(5.00$ g, 42\%).

A solution of $\mathbf{S 3 0}$ (4.0 g, $24 \mathrm{mmol})$ in ether $(25 \mathrm{~mL})$ was added to a suspension of $\mathrm{LiAlH}_{4}(4.6 \mathrm{~g}, 120 \mathrm{mmol})$ in ether $(50 \mathrm{~mL})$ at $0^{\circ} \mathrm{C}$. The resulting mixture warmed to 
room temperature over $3 \mathrm{~h}$. The resulting suspension was cooled to $0{ }^{\circ} \mathrm{C}$, diluted with ether $(200 \mathrm{~mL})$, and quenched by slow addition of $6 \mathrm{M} \mathrm{NaOH}(100 \mathrm{~mL})$. The resulting mixture was extracted with ether $(3 \times 100 \mathrm{~mL})$ and the combined ether extracts were dried $\left(\mathrm{MgSO}_{4}\right)$ and concentrated to give 1-(1-aminomethylcyclohexyl)-2-propen-1-ol (S31) $(2.78 \mathrm{~g}, 68 \%)$.

A solution of $\mathbf{S 3 1}(2.23 \mathrm{~g}, 13.2 \mathrm{mmol})$ and benzaldehyde $(1.39 \mathrm{~g}, 13.1 \mathrm{mmol})$ in $\mathrm{MeOH}(30 \mathrm{~mL})$ was stirred at room temperature for $2 \mathrm{~h}$, treated with $\mathrm{NaBH}_{4}(1.01 \mathrm{~g}, 26.7$ mmol) and stirred overnight. Water $(75 \mathrm{~mL})$ and $1 \mathrm{M} \mathrm{NaOH}(25 \mathrm{~mL})$ were added sequentially and the resulting mixture was extracted with ether $(4 \times 100 \mathrm{~mL})$. The combined ether extracts were washed with a mixture of brine and $1 \mathrm{M} \mathrm{NaOH}[3 / 1(\mathrm{v} / \mathrm{v})$, $100 \mathrm{~mL}]$, dried $\left(\mathrm{MgSO}_{4}\right)$, and concentrated. The resulting oily residue was chromatographed $\left(\mathrm{CH}_{2} \mathrm{Cl}_{2}-\mathrm{MeOH}=20: 1\right)$ to give 1-[1-(benzylaminomethyl)cyclohexyl]-2-propen-1-ol (S32) (2.41 g, 71\%).

A solution of $\mathbf{S 3 2}$ (1.66 g, $6.4 \mathrm{mmol}), t$-butyldimethylsilyl chloride (1.49 g, 9.9 mmol), $\mathrm{Et}_{3} \mathrm{~N}(0.90 \mathrm{~mL}, 6.4 \mathrm{mmol})$, and DMAP (780 mg, $\left.6.4 \mathrm{mmol}\right)$ in $\mathrm{CH}_{2} \mathrm{Cl}_{2}(120 \mathrm{~mL})$ was stirred for $22 \mathrm{~h}$ at room temperature. Water $(70 \mathrm{~mL})$ and $1 \mathrm{M} \mathrm{NaOH}(30 \mathrm{~mL})$ were added sequentially, the layers were separated, and the $\mathrm{CH}_{2} \mathrm{Cl}_{2}$ layer was dried $\left(\mathrm{MgSO}_{4}\right)$ and concentrated. The resulting oily residue was chromatographed (hexanes-EtOAc $=$ 3:1) to give $\mathbf{S 3 3}(2.00 \mathrm{~g}, 84 \%)$.

For S30: TLC (hexanes-EtOAc $=5: 1): \mathrm{R}_{f}=0.18 .{ }^{1} \mathrm{H}$ NMR: $\delta 6.00(\mathrm{ddd}, J=$ 7.2, 10.4, $17.1 \mathrm{~Hz}, 1 \mathrm{H}), 5.35-5.40(\mathrm{~m}, 2 \mathrm{H}), 3.92(\mathrm{~d}, J=7.2 \mathrm{~Hz}, 1 \mathrm{H}), 2.16-2.22(\mathrm{~m}, 1$ H), 1.56-1.88 (m, $7 \mathrm{H}), 1.10-1.36(\mathrm{~m}, 3 \mathrm{H}) .{ }^{13} \mathrm{C}\left\{{ }^{1} \mathrm{H}\right\} \mathrm{NMR}: \delta$ 135.5, 122.1, 119.6, 78.0, 
45.0, 31.8, 31.6, 25.4, 22.8. IR (neat, $\mathrm{cm}^{-1}$ ): 3457, 2934, 2861, 2236, 1450, 933. Anal. calcd (found) for $\mathrm{C}_{10} \mathrm{H}_{15} \mathrm{NO}$ : H, 9.15 (9.30); C, 72.69 (72.95); N, 8.48 (8.52).

For S31： $\mathrm{TLC}\left(\mathrm{CH}_{2} \mathrm{Cl}_{2}-\mathrm{MeOH}=85: 15\right): \mathrm{R}_{f}=0.36 .{ }^{1} \mathrm{H}$ NMR: $\delta \quad 5.96-6.04(\mathrm{~m}$, $1 \mathrm{H}), 5.18-5.34(\mathrm{~m}, 2 \mathrm{H}), 4.08(\mathrm{~d}, J=6.2 \mathrm{~Hz}, 1 \mathrm{H}), 2.74-3.15(\mathrm{~m}, 4 \mathrm{H}), 1.21-1.75(\mathrm{~m}, 11$ H). ${ }^{13} \mathrm{C}\left\{{ }^{1} \mathrm{H}\right\}$ NMR: $\delta \quad 138.6,115.7,80.4,46.7,38.8,31.2,30.8,26.4,21.5,21.4$. IR (neat, $\mathrm{cm}^{-1}$ ): 3371, 2924, 2856, 1597, 1453, 916. Anal. calcd (found) for $\mathrm{C}_{10} \mathrm{H}_{19} \mathrm{NO}: \mathrm{H}$, 11.31 (11.37); C, 70.96 (70.87); N, 8.28 (8.33).

For S32: $\mathrm{TLC}\left(\mathrm{CH}_{2} \mathrm{Cl}_{2}-\mathrm{MeOH}=20: 1\right): \mathrm{R}_{f}=0.27 .{ }^{1} \mathrm{H}$ NMR: $\delta$ 7.25-7.37 $(\mathrm{m}, 5$ H), 5.90 (ddd, $J=6.2,10.6,17.1 \mathrm{~Hz}, 1 \mathrm{H}), 5.26-5.31(\mathrm{~m}, 1 \mathrm{H}), 5.14-5.18(\mathrm{~m}, 1 \mathrm{H}), 4.05-$ 4.07 (m, $1 \mathrm{H}), 3.73(\mathrm{~s}, 2 \mathrm{H}), 2.76(\mathrm{~d}, J=12.3 \mathrm{~Hz}, 1 \mathrm{H}), 2.64-2.67(\mathrm{~m}, 1 \mathrm{H}), 1.22-1.73(\mathrm{~m}$, $12 \mathrm{H}) .{ }^{13} \mathrm{C}\left\{{ }^{1} \mathrm{H}\right\}$ NMR: $\delta$ 139.2, 138.4, 128.7, 128.4, 127.5, 115.9, 80.3, 54.6, 54.3, 39.3, 31.5, 26.4, 21.5, 21.4. IR (neat, $\mathrm{cm}^{-1}$ ): 3283, 2925, 2854, 1451, 919, 697. Anal. calcd (found) for $\mathrm{C}_{17} \mathrm{H}_{25} \mathrm{NO}: \mathrm{H}, 9.71$ (9.71); C, 78.72 (78.61); N, 5.40 (5.58).

For S33: TLC (hexanes-EtOAc $=6: 1): \mathrm{R}_{f}=0.59 .{ }^{1} \mathrm{H}$ NMR (Figure S5): $\delta$ 7.19-7.35 (m, $5 \mathrm{H}), 5.69-5.78(\mathrm{~m}, 1 \mathrm{H}), 5.03-5.08(\mathrm{~m}, 2 \mathrm{H}), 3.93(\mathrm{~d}, J=7.5 \mathrm{~Hz}, 1 \mathrm{H})$, $3.78(\mathrm{~d}, J=13.5 \mathrm{~Hz}, 1 \mathrm{H}), 3.68(\mathrm{~d}, J=13.5 \mathrm{~Hz}, 1 \mathrm{H}), 2.51-2.58(\mathrm{~m}, 2 \mathrm{H}), 1.85(\mathrm{br} \mathrm{s}, 1$ H), 1.70-1.74 (m, 1 H), 1.13-1.56 (m, 9 H), 0.86 (s, 9 H), 0.03 (s, 3 H), -0.02 (s, 3 H). ${ }^{13} \mathrm{C}\left\{{ }^{1} \mathrm{H}\right\}$ NMR (Figure S6): $\delta 141.3,138.7,128.3,128.2,126.7,116.1,81.2,54.9,51.6$, $40.8,31.5,30.7,26.5,26.1,21.8,21.7,18.3,-3.8,-4.9$. IR (neat, $\mathrm{cm}^{-1}$ ): 2928, 2856, 1459, 1065, 836, 774. HRMS calcd (found) for $\mathrm{C}_{23} \mathrm{H}_{39} \mathrm{NOSi}\left(\mathrm{M}^{+}\right)$: 373.2801 (373.2795).

\section{Optimization of Catalytic Hydroamination}


The conditions for the platinum-catalyzed hydroamination of benzyl(2,2dimethyl-4-pentenyl)amine (S11) were optimized for ligand and solvent (Table S1).

Table S1. Effect of ligand and solvent on the reaction of S11 $(0.50 \mathrm{M})$ with a catalytic mixture of $2(2.5 \mathrm{~mol} \%)$ and ligand $(5 \mathrm{~mol} \%)$ at $120^{\circ} \mathrm{C}$.

\begin{tabular}{cccccc}
\hline entry & ligand & solvent & $\mathrm{t}(\mathrm{h})$ & ${\text { conversion }(\%)^{a}}^{\text {yield }(\%)^{a}}$ \\
\hline 1 & $\mathrm{PPh}_{3}$ & 1,4-Dioxane & 9 & $>95$ & 88 \\
2 & $\mathrm{P}\left(4-\mathrm{C}_{6} \mathrm{H}_{4} \mathrm{OMe}\right)_{3}$ & 1,4-Dioxane & 18 & 95 & 40 \\
3 & $\left.\mathrm{P}_{4}-\mathrm{C}_{6} \mathrm{H}_{4} \mathrm{CF}_{3}\right)_{3}$ & 1,4-Dioxane & 18 & 95 & 67 \\
4 & $\mathrm{P}^{\left(\mathrm{OPh}_{3}\right)_{3}}$ & 1,4-Dioxane & 18 & 57 & 32 \\
5 & $\mathrm{PPh}_{3}$ & THF & 9 & $>95$ & 85 \\
6 & $\mathrm{PPh}_{3}$ & i-PrOH & 9 & $>95$ & 72 \\
7 & $\mathrm{PPh}_{3}$ & $\mathrm{CHCl}$ & 19 & 69 & 31 \\
8 & $\mathrm{PPh}_{3}$ & $\mathrm{DCE}$ & 9 & $>95$ & 67 \\
9 & $\mathrm{PPh}_{3}$ & Benzene & 19 & $>95$ & 80 \\
\hline
\end{tabular}

${ }^{a}$ Conversion and yield determined by GC versus $n$-hexadecane internal standard.

\section{Nitrogen Heterocycles}

1-Benzyl-2,4,4-trimethyl-pyrrolidine (Table 1, entry 1; S34), 1-(2benzyloxyethyl)-2,4,4-trimethyl-pyrrolidine (Table 1, entry 2; S35), 1-cyclohexyl-2,4,4trimethyl-pyrrolidine (Table 1, entry 3; S36), 2-benzyl-3,3-dimethyl-2-azaspiro[4.5]decane (Table 1, entry 6; S38), 2-benzyl-4-(tert-butyldimethylsilyloxy)-3methyl-2-aza-spiro[4.5]decane (Table 1, entry 7; S39), and 1-benzyl-4-(4methoxyphenyl)-2-methyl-pyrrolidine (Table 1, entry 9; S41) were synthesized in a manner similar to that described for the synthesis of 2-benzyl-3-methyl-2-aza- 
spiro[4.5]decane (Table 1, entry 4; S37) (see below). 1-Benzyl-4-isopropyl-2-methylpyrrolidine (Table 1, entry 8; S40), 1-(4-bromobenzyl)-2-methyl-4,4-diphenylpyrrolidine (Table 1, entry 10; S42), 4-(2-methyl-4,4-diphenyl-pyrrolidin-1-ylmethyl)benzonitrile (Table 1, entry 11; S 4 3), 2-methyl-1-(4-nitrobenzyl)-4,4-diphenylpyrrolidine (Table 1, entry 12; S44), methyl 4-(2-methyl-4,4-diphenyl-pyrrolidin-1ylmethyl)-benzoate (Table 1, entry 13; S45), and 1-benzo[1,3]dioxol-5-ylmethyl-2methyl-4,4-diphenyl-pyrrolidine (Table 1, entry 14; S46) were synthesized in manner similar to that described for the synthesis of $\mathbf{3}$ (see below). Reaction times and yields are given in Table 1.

1-Benzyl-2-methyl-4,4-diphenyl-pyrrolidine (3). Compound 1 (168 mg, 0.51 mmol) and $n$-hexadecane (42 $\mathrm{mg}$, internal standard) were added to a light yellow suspension of $2(7.4 \mathrm{mg}, 0.013 \mathrm{mmol})$ and $\mathrm{PPh}_{3}(6.6 \mathrm{mg}, 0.025 \mathrm{mmol})$ in dioxane $(1 \mathrm{~mL})$. The reaction vessel was evacuated at $77 \mathrm{~K}$, backfilled with $\mathrm{N}_{2}$, sealed at $77 \mathrm{~K}$, and heated at $120{ }^{\circ} \mathrm{C}$ with stirring for $16 \mathrm{~h}$ (calculated pressure $\approx 5 \mathrm{~atm}$ ). The resulting mixture was diluted with EtOAc $(3 \mathrm{~mL})$, treated with $\mathrm{Al}_{2} \mathrm{O}_{3}(900 \mathrm{mg})$, and concentrated under vacuum. The resulting impregnated $\mathrm{Al}_{2} \mathrm{O}_{3}$ was dry-loaded and chromatographed $\left(\mathrm{Al}_{2} \mathrm{O}_{3}\right.$; hexanes-EtOAc $=200: 1)$ to give $3(125.3 \mathrm{mg}, 75 \%)$ as a colorless oil that solidified to a white solid upon standing. $\mathrm{mp} 57-59{ }^{\circ} \mathrm{C} .{ }^{1} \mathrm{H}$ NMR: $\delta$ 7.13-7.43 (m, $\left.15 \mathrm{H}\right), 4.14(\mathrm{~d}, J=$ 13.2 Hz, $1 \mathrm{H}), 3.69$ (d, $J=9.7 \mathrm{~Hz}, 1 \mathrm{H}), 3.30(\mathrm{~d}, J=13.2 \mathrm{~Hz}, 1 \mathrm{H}), 2.94-2.99(\mathrm{~m}, 1 \mathrm{H})$, 2.82-2.91 (m, $2 \mathrm{H}), 2.26(\mathrm{dd}, J=7.7 \mathrm{~Hz}, 12.6,1 \mathrm{H}), 1.21(\mathrm{~d}, J=6.0 \mathrm{~Hz}, 3 \mathrm{H}) .{ }^{13} \mathrm{C}\left\{{ }^{1} \mathrm{H}\right\}$ NMR: $\delta 150.9,148.9,140.3,128.8,128.4,128.3,128.0,127.6,127.4,127.0,126.0$, 125.6, 66.6, 59.9, 58.2, 52.7, 48.2, 19.7. IR (neat, $\mathrm{cm}^{-1}$ ): 3056, 2961, 2790, 1445, 696. Anal. calcd (found) for $\mathrm{C}_{24} \mathrm{H}_{25} \mathrm{~N}$ : H, 7.70 (7.74); C, 88.03 (88.04); N, 4.28 (4.25). 
For S34: ${ }^{\mathrm{S} 5}$ TLC (hexanes-EtOAc $\left.=8: 1\right): \mathrm{R}_{f}=0.39 .{ }^{1} \mathrm{H}$ NMR: $\delta$ 7.16-7.37 $(\mathrm{m}$, $5 \mathrm{H}), 4.01(\mathrm{~d}, J=13.2 \mathrm{~Hz}, 1 \mathrm{H}), 3.11(\mathrm{~d}, J=13.2 \mathrm{~Hz}, 1 \mathrm{H}), 2.64(\mathrm{~d}, J=9.2 \mathrm{~Hz}, 1 \mathrm{H}), 2.56$ (m, $1 \mathrm{H}), 1.94(\mathrm{~d}, J=9.2 \mathrm{~Hz}, 1 \mathrm{H}), 1.69-1.74(\mathrm{~m}, 1 \mathrm{H}), 1.29-1.35(\mathrm{~m}, 1 \mathrm{H}), 1.15(\mathrm{~d}, J=$ 6.0, $3 \mathrm{H}), 1.06(\mathrm{~s}, 3 \mathrm{H}), 0.97$ (s, $3 \mathrm{H}) .{ }^{13} \mathrm{C}\left\{{ }^{1} \mathrm{H}\right\}$ NMR: $\delta$ 140.0, 128.9, 128.2, 126.8, 68.4, 60.0, 58.1, 49.2, 35.5, 30.7, 29.4, 19.53. IR (neat, $\mathrm{cm}^{-1}$ ): 2954, 2784, 1453, 1372, 736, 697. HRMS calcd (found) for $\mathrm{C}_{14} \mathrm{H}_{21} \mathrm{~N}\left(\mathrm{M}^{+}\right)$: 203.1674 (203.1674). Rapid decomposition of $\mathbf{S 3 4}$ in solution ( $2 \%$ decomposition in $30 \mathrm{~min})$ combined with the high volatility of $\mathbf{S 3 4}$ precluded the isolation of analytically or spectroscopically pure samples of S34.

For S35: ${ }^{1} \mathrm{H}$ NMR (Figure S7): $\delta$ 7.23-7.32 (m, $\left.5 \mathrm{H}\right), 4.52(\mathrm{~s}, 2 \mathrm{H}), 3.52-3.56$ (m, $2 \mathrm{H}), 2.98-3.05(\mathrm{~m}, 1 \mathrm{H}), 2.87$ (d, $J=9.2 \mathrm{~Hz}, 1 \mathrm{H}), 2.41-2.49(\mathrm{~m}, 1 \mathrm{H}), 2.23-2.29(\mathrm{~m}$, $1 \mathrm{H}), 2.01(\mathrm{~d}, J=9.2 \mathrm{~Hz}, 1 \mathrm{H}), 1.61-1.66(\mathrm{~m}, 1 \mathrm{H}), 1.23-1.26(\mathrm{~m}, 1 \mathrm{H}), 1.08(\mathrm{~s}, 3 \mathrm{H})$, $1.06(\mathrm{~d}, J=6.2 \mathrm{~Hz}, 3 \mathrm{H}), 0.98(\mathrm{~s}, 3 \mathrm{H}) .{ }^{13} \mathrm{C}\left\{{ }^{1} \mathrm{H}\right\}$ NMR (Figure S8): $\delta$ 138.6, 128.4, $127.8,127.6,73.2,69.4,69.4,60.7,53.7,49.0,35.7,30.7,29.7,19.2$. IR (neat, $\mathrm{cm}^{-1}$ ): 2952, 2864, 2787, 1103, 735, 696. TLC (hexanes-EtOAc $=2: 1$ ): $\mathrm{R}_{f}=0.38$. HRMS calcd (found) for $\mathrm{C}_{16} \mathrm{H}_{25} \mathrm{NO}\left(\mathrm{M}^{+}\right)$: 247.1936 (247.1933).

For S36: $\quad \mathrm{TLC}\left(\mathrm{CH}_{2} \mathrm{Cl}_{2}-\mathrm{MeOH}=9: 1\right): \quad \mathrm{R}_{f}=0.11 .{ }^{1} \mathrm{H}$ NMR (Figure S9): $\delta$ 2.89-2.97 (m, $1 \mathrm{H}), 2.65$ (d, $J=9.1 \mathrm{~Hz}, 1 \mathrm{H}), 2.48-2.56(\mathrm{~m}, 1 \mathrm{H}), 2.37$ (d, $J=9.1 \mathrm{~Hz}, 1$ H), 1.70-1.80 (m, 4 H), 1.57-1.66 (m, 2 H), 1.10-1.30 (m, 6 H), 1.08 (s, 3 H), 1.03 (d, $J=$ $6.2 \mathrm{~Hz}, 3 \mathrm{H}), 0.97(\mathrm{~s}, 3 \mathrm{H}) .{ }^{13} \mathrm{C}\left\{{ }^{1} \mathrm{H}\right\}$ NMR (Figure S10): $\delta$ 61.8, 57.2, 54.5, 49.1, 35.4, 33.4, 30.2, 29.6, 26.6, 26.5, 25.8, 24.9, 19.6. IR (neat, $\mathrm{cm}^{-1}$ ): 2928, 2857, 1451, 1372. HRMS calcd (found) for $\mathrm{C}_{13} \mathrm{H}_{25} \mathrm{~N}\left(\mathrm{M}^{+}\right)$: 195.1987 (195.1989). 
2-Benzyl-3-methyl-2-aza-spiro[4.5]decane (S37). ${ }^{\text {S5 }}$ A suspension of $\mathbf{S 2 2}(124$ mg, $0.509 \mathrm{mmol}), n$-hexadecane (27 mg, $0.12 \mathrm{mmol}), 2$ (7.4 mg, $0.013 \mathrm{mmol})$, and $\mathrm{PPh}_{3}$ (6.6 mg, $0.025 \mathrm{mmol})$ in dioxane $(1 \mathrm{~mL})$ was heated at $120^{\circ} \mathrm{C} 2 \mathrm{~h}$. The resulting mixture was filtered through a short plug of Celite, which was eluted with $\mathrm{CH}_{2} \mathrm{Cl}_{2}(3 \mathrm{~mL})$. The resulting solution was treated with acetic anhydride $(1 \mathrm{~mL}, 10 \mathrm{mmol})$, stirred for $2.5 \mathrm{~h}$, and concentrated under vacuum. The resulting residue was dissolved in ether/EtOAc $(1 / 1 ; 4 \mathrm{~mL})$ and extracted with $1 \mathrm{M} \mathrm{HCl}(5 \times 1.5 \mathrm{~mL})$. The combined aqueous extracts were basified $(\mathrm{pH}>12)$ with $6 \mathrm{M} \mathrm{NaOH}$ and extracted with $\mathrm{CH}_{2} \mathrm{Cl}_{2}(5 \times 3 \mathrm{~mL})$. The combined $\mathrm{CH}_{2} \mathrm{Cl}_{2}$ extracts were dried $\left(\mathrm{MgSO}_{4}\right)$, filtered through a short plug of $\mathrm{Al}_{2} \mathrm{O}_{3}$, which was eluted with EtOAc ( $5 \mathrm{~mL}$ ), and concentrated to give pure $\mathbf{S 3 7}$ as a pale yellow oil $(102 \mathrm{mg}, 82 \%) . \quad$ TLC (hexanes-EtOAc $=6: 1): \mathrm{R}_{f}=0.45 .{ }^{1} \mathrm{H}$ NMR: $\delta 7.21-7.34(\mathrm{~m}$, $5 \mathrm{H}), 4.01(\mathrm{~d}, J=13.3 \mathrm{~Hz}, 1 \mathrm{H}), 3.09(\mathrm{~d}, J=13.3 \mathrm{~Hz}, 1 \mathrm{H}), 2.77(\mathrm{~d}, J=9.2 \mathrm{~Hz}, 1 \mathrm{H})$, 2.44-2.52 (m, $1 \mathrm{H}), 1.86(\mathrm{~d}, J=9.2 \mathrm{~Hz}, 1 \mathrm{H}), 1 \mathrm{H}(\mathrm{dd}, J=7.0,12.5 \mathrm{~Hz}, 1 \mathrm{H}), 1.25-1.45$ $(\mathrm{m}, 11 \mathrm{H}), 1.14(\mathrm{~d}, J=6.0 \mathrm{~Hz}, 3 \mathrm{H}) .{ }^{13} \mathrm{C}\left\{{ }^{1} \mathrm{H}\right\} \mathrm{NMR}: \delta 140.1,128.8,128.2,126.7,66.8$, 59.1, 58.1, 47.1, 39.4, 38.6, 26.2, 23.8, 23.6, 19.4. IR (neat, $\mathrm{cm}^{-1}$ ): 2922, 2851, 2783, 1449, 1373, 735. Anal. calcd (found) for $\mathrm{C}_{17} \mathrm{H}_{25} \mathrm{~N}$ : H, 10.35 (10.54); C, 83.89 (83.77); N, 5.75 (5.92).

For S38: ${ }^{\mathrm{S} 5}$ TLC (hexanes-EtOAc $\left.=5: 1\right): \mathrm{R}_{f}=0.74 .{ }^{1} \mathrm{H}$ NMR: $\delta \quad 7.18-7.36(\mathrm{~m}$, $5 \mathrm{H}), 3.50(\mathrm{~s}, 2 \mathrm{H}), 2.41(\mathrm{~s}, 2 \mathrm{H}), 1.55(\mathrm{~s}, 2 \mathrm{H}), 1.26-1.42(\mathrm{~m}, 10 \mathrm{H}), 1.08(\mathrm{~s}, 6 \mathrm{H})$. ${ }^{13} \mathrm{C}\left\{{ }^{1} \mathrm{H}\right\}$ NMR: $\delta \quad 141.8,128.2,128.1,126.4,62.8,60.1,53.9,52.2,39.3,39.1,26.2$, 24.8, 23.9. IR (neat, $\mathrm{cm}^{-1}$ ): 2923, 2852, 1449, 1361, 729, 696. Anal. calcd (found) for $\mathrm{C}_{18} \mathrm{H}_{27} \mathrm{~N}: \mathrm{H}, 10.57$ (10.34); C, 83.99 (83.74); N, 5.44 (5.61). 
For S39: The 3.5:1 diastereotopic mixture obtained via chromatography was chromatographed a second time $\left(\mathrm{Al}_{2} \mathrm{O}_{3} ;\right.$ hexanes-EtOAc $\left.=100: 1\right)$ to produce two fractions, one enriched in the minor diastereomer (2:1), and one enriched in the major diastereomer (39:1), which facilitated identification of the ${ }^{1} \mathrm{H}$ and ${ }^{13} \mathrm{C}$ NMR (Figures S11, S12) resonances corresponding to the major and minor isomers of S39. ${ }^{1} \mathrm{H}$ NMR (major diastereomer): $\delta$ 7.22-7.32 (m, $5 \mathrm{H}), 4.00(\mathrm{~d}, J=13.2 \mathrm{~Hz}, 1 \mathrm{H}), 3.30(\mathrm{~d}, J=5.5 \mathrm{~Hz}, 1$ H), $3.16(\mathrm{~d}, J=12.6 \mathrm{~Hz}, 1 \mathrm{H}), 2.86(\mathrm{~d}, J=8.9 \mathrm{~Hz}, 1 \mathrm{H}), 2.32-2.38(\mathrm{~m}, 1 \mathrm{H}), 2.05(\mathrm{~d}, J=$ $8.2 \mathrm{~Hz}, 1 \mathrm{H}), 1.07-1.53(\mathrm{~m}, 13 \mathrm{H}), 0.91(\mathrm{~s}, 9 \mathrm{H}), 0.09$ (s, $3 \mathrm{H}), 0.08$ (s, $3 \mathrm{H}) .{ }^{1} \mathrm{H}$ NMR (minor diastereomer): $\delta$ 7.21-7.32 (m, $5 \mathrm{H}), 3.88(\mathrm{~d}, J=13.7 \mathrm{~Hz}, 1 \mathrm{H}), 3.67(\mathrm{~d}, J=7.3$ $\mathrm{Hz}, 1 \mathrm{H}), 3.39$ (d, $J=14.2 \mathrm{~Hz}, 1 \mathrm{H}), 2.76-2.88(\mathrm{~m}, 1 \mathrm{H}), 1.97$ (d, $J=7.5 \mathrm{~Hz}, 1 \mathrm{H}), 1.67$ (d, $J=12.6 \mathrm{~Hz}, 1 \mathrm{H}), 0.98-1.66(\mathrm{~m}, 13 \mathrm{H}), 0.94(\mathrm{~s}, 9 \mathrm{H}), 0.06(\mathrm{~s}, 3 \mathrm{H}), 0.04(\mathrm{~s}, 3 \mathrm{H})$. ${ }^{13} \mathrm{C}\left\{{ }^{1} \mathrm{H}\right\}$ NMR (major diastereomer): $\delta \quad 139.9,128.8,128.3,126.8,86.8,66.0,62.2$, 58.1, 42.9, 36.4, 32.2, 26.4, 26.1, 23.9, 23.0, 18.3, 17.7, -3.7, -3.9. ${ }^{13} \mathrm{C}\left\{{ }^{1} \mathrm{H}\right\}$ NMR (minor diastereomer): $\delta 140.1,128.6,128.3,126.8,81.6,62.2,59.4,58.0,45.2,37.0$, $30.2,26.5,26.2,24.2,23.3,18.4,17.7,-4.2$. IR $\left(\right.$ neat, $\mathrm{cm}^{-1}$ ): 2929, 2856, 1665, 1452, 1252, 835. TLC (hexanes-EtOAc $=8: 1): \quad R_{f}=0.50$. HRMS calcd (found) for $\mathrm{C}_{23} \mathrm{H}_{40} \mathrm{NOSi}\left(\mathrm{MH}^{+}\right): 374.2879$ (374.2876).

For S40: The purified mixture of diastereomers was chromatographed a second time $\left(\mathrm{Al}_{2} \mathrm{O}_{3}\right.$; hexanes-EtOAc $\left.=100: 1\right)$ to give $\mathbf{S 4 0}$ enriched in the major diastereomer (10:1), which facilitated identification of the ${ }^{1} \mathrm{H}$ and ${ }^{13} \mathrm{C}$ NMR resonances (Figures S13, S14) corresponding to the major and minor isomers of S40. ${ }^{1} \mathrm{H}$ NMR (major diastereomer): $\delta$ 7.22-7.35 (m, $5 \mathrm{H}), 3.99(\mathrm{~d}, J=12.8 \mathrm{~Hz}, 1 \mathrm{H}), 3.13(\mathrm{~d}, J=12.8 \mathrm{~Hz}, 1$ H), 2.95-3.01 (m, $1 \mathrm{H}), 2.41-2.50(\mathrm{~m}, 1 \mathrm{H}), 1.57-1.84(\mathrm{~m}, 4 \mathrm{H}), 1.30-1.41(\mathrm{~m}, 1 \mathrm{H}), 1.16$ 
(d, $J=5.8 \mathrm{~Hz}, 3 \mathrm{H}), 0.84-0.87(\mathrm{~m}, 6 \mathrm{H}) .{ }^{1} \mathrm{H}$ NMR (minor diastereomer): $\delta \quad 7.22-7.35$ (m, $5 \mathrm{H}), 4.01(\mathrm{~d}, J=13.3 \mathrm{~Hz}, 1 \mathrm{H}), 3.22(\mathrm{~d}, J=12.6 \mathrm{~Hz}, 1 \mathrm{H}), 2.71-2.76(\mathrm{~m}, 1 \mathrm{H}), 2.54-$ $2.59(\mathrm{~m}, 1 \mathrm{H}), 2.38(\mathrm{t}, J=9.6 \mathrm{~Hz}, 1 \mathrm{H}), 1.95-2.02(\mathrm{~m}, 1 \mathrm{H}), 1.65-1.75(\mathrm{~m}, 1 \mathrm{H}), 1.40-1.49$ (m, $1 \mathrm{H}), 1.18-1.26(\mathrm{~m}, 1 \mathrm{H}), 1.17(\mathrm{~d}, J=6.0 \mathrm{~Hz}, 3 \mathrm{H}), 0.84(\mathrm{~d}, J=6.5 \mathrm{~Hz}, 3 \mathrm{H}), 0.80(\mathrm{~d}$, $J=6.7 \mathrm{~Hz}, 3 \mathrm{H}$ ). ${ }^{13} \mathrm{C}\left\{{ }^{1} \mathrm{H}\right\}$ NMR (major diastereomer): $\delta$ 139.7, 129.0, 128.3, 127.0, 60.4, 58.1, 57.8, 43.2, 39.1, 33.2, 21.5, 20.8, 18.8. ${ }^{13} \mathrm{C}\left\{{ }^{1} \mathrm{H}\right\}$ NMR (minor diastereomer): $\delta 140.1,128.9,128.2,126.7,59.8,59.6,58.8,43.2,38.1,33.1,21.5,21.2,20.0$. IR (neat, $\mathrm{cm}^{-1}$ ): 2957, 2871, 2785, 1373, 736, 697. TLC (hexanes-EtOAc $\left.=2: 1\right): \quad \mathrm{R}_{f}=0.70$. HRMS calcd (found) for $\mathrm{C}_{15} \mathrm{H}_{23} \mathrm{~N}\left(\mathrm{M}^{+}\right)$: 217.1830 (217.1834).

For S41: Pale yellow oil. The purified mixture of diastereomers was chromatographed a second time $\left(\mathrm{Al}_{2} \mathrm{O}_{3} ;\right.$ hexanes-EtOAc $\left.=200: 1 \rightarrow 50: 1\right)$ to give $\mathbf{S 4 1}$ enriched in the major diastereomer (8:1), which facilitated identification of the ${ }^{1} \mathrm{H}$ and ${ }^{13} \mathrm{C}$ NMR (Figures S15, S16) resonances corresponding to the major and minor isomers of S41. TLC (hexanes-EtOAc $=5: 1$ ): $\mathrm{R}_{f}=0.21 .{ }^{1} \mathrm{H}$ NMR (major diastereomer): $\delta$ 7.22-7.38 (m, $5 \mathrm{H}), 7.12-7.15(\mathrm{~m}, 2 \mathrm{H}), 6.82-6.85(\mathrm{~m}, 2 \mathrm{H}), 4.07(\mathrm{~d}, J=12.6 \mathrm{~Hz}, 1 \mathrm{H})$, $3.78(\mathrm{~s}, 3 \mathrm{H}), 3.21-3.53(\mathrm{~m}, 3 \mathrm{H}), 2.73-2.82(\mathrm{~m}, 1 \mathrm{H}), 2.21(\mathrm{t}, J=9.2 \mathrm{~Hz}, 1 \mathrm{H}), 1.95-2.12$ $(\mathrm{m}, 2 \mathrm{H}), 1.26(\mathrm{~d}, J=6.0 \mathrm{~Hz}, 3 \mathrm{H}) .{ }^{1} \mathrm{H}$ NMR (minor diastereomer): $\delta \quad 7.21-7.40(\mathrm{~m}, 7$ H), 6.80-6.83 (m, $2 \mathrm{H}), 4.12(\mathrm{~d}, J=13.3 \mathrm{~Hz}, 1 \mathrm{H}), 3.77(\mathrm{~s}, 3 \mathrm{H}), 3.28(\mathrm{~d}, J=12.6 \mathrm{~Hz}, 1$ H), 3.12-3.20 (m, $1 \mathrm{H}), 2.98(\mathrm{dd}, J=4.8,9.9 \mathrm{~Hz}, 1 \mathrm{H}), 2.64-2.72(\mathrm{~m}, 2 \mathrm{H}), 2.41$ (ddd, $J=$ 6.2, 8.7, $12.5 \mathrm{~Hz}, 1 \mathrm{H}), 1.54-1.64(\mathrm{~m}, 1 \mathrm{H}), 1.26(\mathrm{~d}, J=5.8 \mathrm{~Hz}, 3 \mathrm{H}) .{ }^{13} \mathrm{C}\left\{{ }^{1} \mathrm{H}\right\} \mathrm{NMR}$ (major diastereomer): $\delta 158.0,136.5,129.0,128.4,127.1,113.9,62.9,60.3,58.4,55.4$, 41.3, 40.6, 19.8. ${ }^{13} \mathrm{C}\left\{{ }^{1} \mathrm{H}\right\}$ NMR (minor diastereomer): $\delta$ 158.2, 137.5, 129.4, 128.6, 
128.3, 127.7, 114.0, 61.4, 60.8, 57.7, 55.4, 43.5, 40.7, 18.1. IR (neat, $\mathrm{cm}^{-1}$ ): 2956, 2788, 1512, 1245, 1034, 827. HRMS calcd (found) for $\mathrm{C}_{19} \mathrm{H}_{23} \mathrm{NO}\left(\mathrm{M}^{+}\right)$: 281.1780 (281.1778).

For S42: White solid. $\mathrm{mp} 92-93^{\circ} \mathrm{C} . \quad$ TLC (hexanes-EtOAc $=5: 1$ ): $\mathrm{R}_{f}=0.49$. ${ }^{1} \mathrm{H}$ NMR: $\delta 7.44(\mathrm{~d}, J=8.4 \mathrm{~Hz}, 2 \mathrm{H}), 7.12-7.29(\mathrm{~m}, 12 \mathrm{H}), 4.01(\mathrm{~d}, J=13.3 \mathrm{~Hz}, 1 \mathrm{H})$, $3.60(\mathrm{~d}, J=9.4 \mathrm{~Hz}, 1 \mathrm{H}), 3.22(\mathrm{~d}, J=13.3 \mathrm{~Hz}, 1 \mathrm{H}), 2.89-2.95(\mathrm{~m}, 1 \mathrm{H}), 2.81-2.86(\mathrm{~m}, 1$ H), $2.77(\mathrm{~d}, J=9.9 \mathrm{~Hz}, 1 \mathrm{H}), 2.20-2.25(\mathrm{~m}, 1 \mathrm{H}), 1.16(\mathrm{~d}, J=5.8,3 \mathrm{H}) .{ }^{13} \mathrm{C}\left\{{ }^{1} \mathrm{H}\right\} \mathrm{NMR}$ : $\delta 150.6,148.7,139.4,131.4,130.4,128.3,128.0,127.5,127.3,126.0,125.6,120.6,66.5$, 59.8, 57.4, 52.7, 48.0, 19.6. IR (neat, $\mathrm{cm}^{-1}$ ): 2964, 2797, 1486, 1371, 800, 765. Anal. calcd (found) for $\mathrm{C}_{24} \mathrm{H}_{24} \mathrm{BrN}$ : H, 5.95 (6.23); C, 70.94 (71.05); N, 3.45 (3.60).

For S43: Pale yellow solid. $\mathrm{mp} 101-103{ }^{\circ} \mathrm{C} . \mathrm{TLC}($ hexanes-EtOAc $=4: 1): \mathrm{R}_{f}=$ 0.47. ${ }^{1} \mathrm{H}$ NMR: $\delta 7.58(\mathrm{~d}, J=7.7 \mathrm{~Hz}, 2 \mathrm{H}), 7.43(\mathrm{~d}, J=8.0 \mathrm{~Hz}, 2 \mathrm{H}), 7.14-7.26(\mathrm{~m}, 10$ H), $4.05(\mathrm{~d}, J=14.2 \mathrm{~Hz}, 1 \mathrm{H}), 3.55(\mathrm{~d}, J=9.4 \mathrm{~Hz}, 1 \mathrm{H}), 3.32(\mathrm{~d}, J=13.8 \mathrm{~Hz}, 1 \mathrm{H}), 2.81-$ $2.91(\mathrm{~m}, 2 \mathrm{H}), 2.79$ (d, $J=9.7 \mathrm{~Hz}, 1 \mathrm{H}), 2.21-2.26(\mathrm{~m}, 1 \mathrm{H}), 1.13(\mathrm{~d}, J=5.3 \mathrm{~Hz}, 3 \mathrm{H})$. ${ }^{13} \mathrm{C}\left\{{ }^{1} \mathrm{H}\right\}$ NMR: $\delta \quad 150.2,148.5,146.2,132.3,129.2,128.4,128.1,127.4,127.2,126.1$, $125.8,119.2,110.8,66.6,59.8,57.8,52.9,47.8,19.6$. IR (neat, $\left.\mathrm{cm}^{-1}\right): 2962,2793,1489$, 1242, 1037, 699. Anal. calcd (found) for $\mathrm{C}_{25} \mathrm{H}_{24} \mathrm{~N}_{2}: \mathrm{H}, 6.86$ (6.96); C, 85.19 (85.19); N, 7.95 (8.07).

For S44: Yellow solid. $\mathrm{mp} 127-128{ }^{\circ} \mathrm{C}$. TLC (hexanes-EtOAc $=5: 1$ ): $\mathrm{R}_{f}=$ 0.39. ${ }^{1} \mathrm{H}$ NMR: $\delta 8.18(\mathrm{~d}, J=8.4 \mathrm{~Hz}, 2 \mathrm{H}), 7.52(\mathrm{~d}, J=8.0 \mathrm{~Hz}, 2 \mathrm{H}), 7.15-7.30(\mathrm{~m}, 10$ H), $4.12(\mathrm{~d}, J=14.4 \mathrm{~Hz}, 1 \mathrm{H}), 3.59(\mathrm{~d}, J=9.6 \mathrm{~Hz}, 1 \mathrm{H}), 3.42(\mathrm{~d}, J=14.4 \mathrm{~Hz}, 1 \mathrm{H}), 2.88-$ $2.96(\mathrm{~m}, 2 \mathrm{H}), 2.84(\mathrm{~d}, J=9.6 \mathrm{~Hz}, 1 \mathrm{H}), 2.25-2.32(\mathrm{~m}, 1 \mathrm{H}), 1.18(\mathrm{~s}, 3 \mathrm{H}) .{ }^{13} \mathrm{C}\left\{{ }^{1} \mathrm{H}\right\}$ NMR: $\delta 150.2,148.4,147.2,129.1,128.4,128.1,127.4,127.2,126.1,125.8,123.7$, 
66.6, 59.9, 57.5, 52.9, 47.8, 19.6. IR (neat, $\mathrm{cm}^{-1}$ ): 2963, 2792, 1514, 1341, 702. Anal. calcd (found) for $\mathrm{C}_{24} \mathrm{H}_{24} \mathrm{~N}_{2} \mathrm{O}_{2}$ : H, 6.49 (6.53); C, 77.39 (77.13); N, 7.52 (7.60).

For S45: Pale yellow viscous oil. TLC (hexanes-EtOAc $=4: 1$ ): $\mathrm{R}_{f}=0.53 .{ }^{1} \mathrm{H}$ NMR: $\delta 7.98(\mathrm{~d}, J=8.2 \mathrm{~Hz}, 2 \mathrm{H}), 7.42(\mathrm{~d}, J=7.9 \mathrm{~Hz}, 2 \mathrm{H}), 7.08-7.27(\mathrm{~m}, 10 \mathrm{H}), 4.08$ (d, $J=13.8 \mathrm{~Hz}, 1 \mathrm{H}), 3.90(\mathrm{~s}, 3 \mathrm{H}), 3.59(\mathrm{~d}, J=9.6 \mathrm{~Hz}, 1 \mathrm{H}), 3.30(\mathrm{~d}, J=13.5 \mathrm{~Hz}, 1 \mathrm{H})$, 2.83-2.93 (m, $2 \mathrm{H}), 2.77$ (d, $J=9.2 \mathrm{~Hz}, 1 \mathrm{H}), 2.20-2.25(\mathrm{~m}, 1 \mathrm{H}), 1.15$ (d, $J=5.6 \mathrm{~Hz}, 3$ H). ${ }^{13} \mathrm{C}\left\{{ }^{1} \mathrm{H}\right\}$ NMR: $\delta \quad 167.2,150.4,148.6,145.8,129.7,128.6,128.3,128.0,127.4$, 127.2, 126.0, 125.7, 66.5, 59.9, 57.9, 52.7, 52.2, 52.1, 47.9, 19.6. IR (neat, $\mathrm{cm}^{-1}$ ): 2956, 2798, 1719, 1274, 1105, 697. Anal. calcd (found) for $\mathrm{C}_{26} \mathrm{H}_{27} \mathrm{NO}_{2}: \mathrm{H}, 7.06$ (6.81); C, $81.01(80.95) ; \mathrm{N}, 3.63(3.78)$.

For S46: Viscous colorless oil. TLC (hexanes-EtOAc $=4: 1$ ): $\mathrm{R}_{f}=0.53 .{ }^{1} \mathrm{H}$ NMR: $\delta$ 7.07-7.26 (m, $10 \mathrm{H}), 6.88(\mathrm{~s}, 1 \mathrm{H}), 6.72-6.78(\mathrm{~m}, 2 \mathrm{H}), 5.92-5.93(\mathrm{~m}, 2 \mathrm{H}), 3.95$ (d, $J=13.3 \mathrm{~Hz}, 1 \mathrm{H}), 3.61(\mathrm{~d}, J=9.6 \mathrm{~Hz}, 1 \mathrm{H}), 3.15(\mathrm{~d}, J=12.6 \mathrm{~Hz}, 1 \mathrm{H}), 2.89$ (dd, $J=$ 7.7, $12.6 \mathrm{~Hz}, 1 \mathrm{H}), 2.74-2.81(\mathrm{~m}, 2 \mathrm{H}), 2.15-2.20(\mathrm{~m}, 1 \mathrm{H}), 1.13(\mathrm{~d}, J=5.5 \mathrm{~Hz}, 3 \mathrm{H})$. ${ }^{13} \mathrm{C}\left\{{ }^{1} \mathrm{H}\right\}$ NMR: $\delta 150.7,148.8,147.8,146.5,134.2,128.3,128.0,127.6,127.4,126.0$, 125.6, 121.5, 109.2, 108.0, 101.0, 66.4, 59.6, 57.9, 52.6, 48.1, 19.7. IR (neat, $\mathrm{cm}^{-1}$ ): 2962, 2793, 1489, 1242, 1037, 699. Anal. calcd (found) for $\mathrm{C}_{25} \mathrm{H}_{25} \mathrm{NO}_{2}: \mathrm{H}, 6.78$ (6.87); C, 80.83 (81.11); N, 3.77 (3.96).

2-Benzyl-3-methyl-2-aza-spiro[5.5] undecane (S47; Table 1, entry 15). Amino olefin S24 (129 mg, $0.501 \mathrm{mmol}$ ) was added over $6 \mathrm{~h}$ to a well-stirred suspension 2 (7.4 $\mathrm{mg}, 0.013 \mathrm{mmol})$ and $\mathrm{PPh}_{3}(6.5 \mathrm{mg}, 0.025 \mathrm{mmol})$ in diglyme $(1 \mathrm{~mL})$ at $120^{\circ} \mathrm{C}$. Work-up of the crude reaction mixture as was described for $\mathbf{S 3 7}$ gave $\mathbf{S 4 7}$ (73 $\mathrm{mg}, 57 \%)$. TLC (hexanes-EtOAc $=5: 1): \mathrm{R}_{f}=0.73 .{ }^{1} \mathrm{H}$ NMR: $\delta \quad 7.20-7.35(\mathrm{~m}, 5 \mathrm{H}), 4.00(\mathrm{~d}, J=13.8$ 
$\mathrm{Hz}, 1 \mathrm{H}), 3.05(\mathrm{~d}, J=13.8 \mathrm{~Hz}, 1 \mathrm{H}), 2.52(\mathrm{~d}, J=11.4 \mathrm{~Hz}, 1 \mathrm{H}), 2.21-2.27(\mathrm{~m}, 1 \mathrm{H}), 1.62$ $(\mathrm{d}, J=11.6 \mathrm{~Hz}, 1 \mathrm{H}), 1.04-1.57(\mathrm{~m}, 17 \mathrm{H}) .{ }^{13} \mathrm{C}\left\{{ }^{1} \mathrm{H}\right\} \mathrm{NMR}: \delta \quad 140.8,128.7,128.1$, $126.5,61.8,58.4,57.4,38.1,34.9,33.3,31.0,27.1,21.8,19.3$. IR (neat, $\mathrm{cm}^{-1}$ ): 2922 , 2850, 2783, 1450, 735, 697. Anal. calcd (found) for $\mathrm{C}_{18} \mathrm{H}_{27} \mathrm{~N}$ : H, 10.57 (10.72); C, 83.99 (83.96); N, 5.44 (5.36).

\section{Platinum Complexes}

Addition of 1 (1 equiv) to a $\mathrm{CDCl}_{3}$ solution of $\left[\mathrm{PtCl}_{2} \mathrm{PPh}_{3}\right]_{2}(\mathbf{4}){ }^{\mathrm{S} 10}(33 \mathrm{mM})$ at -20 ${ }^{\circ} \mathrm{C}$ led to rapid $\left(t_{1 / 2} \leq 5 \mathrm{~min}\right)$ formation of the platinum amine complex trans$\mathrm{PtCl}_{2}\left(\mathrm{PPh}_{3}\right)\left[\eta^{1}-\mathrm{NHBnCH}_{2} \mathrm{C}\left(\mathrm{Ph}_{2}\right) \mathrm{CH}_{2} \mathrm{CH}=\mathrm{CH}_{2}\right]\left(\right.$ trans-5) in $97 \pm 5 \%$ yield $\left({ }^{1} \mathrm{H} \mathrm{NMR}\right)$ (Scheme S6). Thermally unstable trans-5 was characterized without isolation by $1 \mathrm{D}{ }^{1} \mathrm{H}$, ${ }^{1} \mathrm{H}\left\{{ }^{31} \mathrm{P}\right\}$, and ${ }^{31} \mathrm{P}$ NMR spectroscopy, by $2 \mathrm{D}{ }^{1} \mathrm{H}-{ }^{1} \mathrm{H} \mathrm{COSY}$ (Figure S17 and S18) and ${ }^{1} \mathrm{H}$

- ${ }^{1} \mathrm{H}$ NOESY (Figure S19 and S20) spectroscopy, and through the synthesis and ${ }^{31} \mathrm{P}\left\{{ }^{1} \mathrm{H}\right\}$ NMR analysis of the ${ }^{15} \mathrm{~N}$-labeled isotopomer trans- $\mathrm{PtCl}_{2}\left(\mathrm{PPh}_{3}\right)\left[\eta^{1}-\right.$ $\left.{ }^{15} \mathrm{NHBnCH}_{2} \mathrm{C}\left(\mathrm{Ph}_{2}\right) \mathrm{CH}_{2} \mathrm{CH}=\mathrm{CH}_{2}\right]$ (trans-5 $-{ }^{15} \mathrm{~N}$ ). The numbering scheme for the aliphatic ${ }^{1} \mathrm{H}$ resonances of trans-5 is depicted in Figure S21. A number of features in the ${ }^{1} \mathrm{H}$ NMR spectrum established trans-5 as a platinum amine complex. These features include the strong upfield shift the of the amine proton of trans-5 ( $\delta 3.81)$ relative to the amine proton of free $1(\delta 0.90)$, the diasterotopic nature of the benzylic [ $\delta 4.52$ (dd, $J=6.6$, $12.7 \mathrm{~Hz}), 3.44(\mathrm{dd}, J=7.3,12.8 \mathrm{~Hz})]$, the $\alpha$-methylene [ $\delta 4.13(\mathrm{dd}, J=5.9,14.1 \mathrm{~Hz}, 1$ $\mathrm{H}), 3.12-3.19(\mathrm{~m}, 1 \mathrm{H})]$, and $\gamma$-methylene [ $\delta 4.57(\mathrm{dd}, J=8.0,12.5 \mathrm{~Hz}), 3.12-3.19(\mathrm{~m}, 1$ $\mathrm{H})$ ] protons of the $\gamma$-amino olefin ligand of trans-5, and the presence of residual coupling between the phosphorous atom and one of the diasterotopic methylene protons $\alpha$ - to the 
amine nitrogen atom $(\delta 4.57)$. The presence of an $\mathrm{N}-\mathrm{P}$ bond and the trans arrangement of the amine and phosphine ligands of trans $-\mathbf{5}$ was firmly established by the large ${ }^{31} \mathrm{P}-{ }^{15} \mathrm{~N}$ coupling constant $\left(J_{\mathrm{PN}}=47 \mathrm{~Hz}\right)$ observed in the ${ }^{31} \mathrm{P}\left\{{ }^{1} \mathrm{H}\right\}$ NMR spectrum of trans-5${ }^{15} \mathrm{~N} .{ }^{\mathrm{S} 11}$ Although the ${ }^{1} \mathrm{H}$ NMR resonance of the internal olefinic proton of trans-5 $(\delta$ 5.94) was shifted downfield relative to the corresponding resonance of free $\mathbf{1}(\delta 5.33)$ olefin coordination in trans-5 was firmly discounted due to the absence of ${ }^{195} \mathrm{Pt}$-satellites for the olefinic resonance of trans-5.

Warming a $\mathrm{CDCl}_{3}$ solution of trans -5 at $20{ }^{\circ} \mathrm{C}$ for $1 \mathrm{~h}$ and $40{ }^{\circ} \mathrm{C}$ for $0.5 \mathrm{~h}$ led to formation of the zwitterionic complex 6 in $94 \pm 5 \%$ yield $\left({ }^{1} \mathrm{H}\right.$ NMR) (Scheme 1$)$. Disappearance of trans -5 at $20{ }^{\circ} \mathrm{C}$ obeyed first-order kinetics to $>2$ half-lives with a rate constant of $k=4.9 \pm 0.3 \times 10^{-4} \mathrm{~s}^{-1}\left(\Delta G^{\ddagger}=21.6 \pm 0.1 \mathrm{kcal} \mathrm{mol}^{-1}\right)$ (Figure S22). Complex 6 was isolated from the corresponding preparative-scale reaction in quantitative $(100 \%)$ yield and was characterized by $1 \mathrm{D}^{1} \mathrm{H}\left\{{ }^{31} \mathrm{P}\right\}$ and ${ }^{31} \mathrm{P}$ NMR spectroscopy, by $2 \mathrm{D}{ }^{1} \mathrm{H}-{ }^{1} \mathrm{H}$ COSY NMR spectroscopy (Figure S23 and S24), and by mass spectrometry. The numbering scheme for the aliphatic ${ }^{1} \mathrm{H}$ resonances of $\mathbf{6}$ is depicted in Figure $\mathrm{S} 25$. The presence of a cationic nitrogen atom in $\mathbf{6}$ was established by the large downfield shift of the ammonium proton of $6(\delta 10.1)$, which exchanged with $\mathrm{D}_{2} \mathrm{O}$, relative to the amine proton of trans-5 $(\delta 3.81)$. Although the ${ }^{195} \mathrm{Pt}$-satellites of the platinum-bound methylene protons were not sufficiently resolved to determine $J_{\mathrm{PtH}}$, the central peak of these resonances integrated to the expected $\sim 0.7 \mathrm{H}$.

Treatment of a dioxane- $d_{8}$ solution of $6(32 \mathrm{mM})$ with $N$-benzyl-4-pentenylamine (7; 5 equiv) at $25^{\circ} \mathrm{C}$ for 5 min led to complete consumption of 6 to form the heterobicyclic platinum amine complex trans-8 (Scheme 1) as determined by ${ }^{1} \mathrm{H}$ NMR 
spectroscopy. Accurate determination of the yield of conversion of $\mathbf{6}$ to trans-8 under these conditions was hindered by the presence of excess 7. Similarly, treatment of 6 with $\mathrm{HNEt}_{2}$ (1 equiv) at room temperature for 5 min led to formation of trans-8 in $104 \pm 5 \%$ yield $\left({ }^{1} \mathrm{H}\right.$ NMR). Complex trans-8 was isolated from the corresponding preparative-scale reaction in $8 \%$ yield and was characterized by $1 \mathrm{D}{ }^{1} \mathrm{H}\left\{{ }^{31} \mathrm{P}\right\}$ and ${ }^{31} \mathrm{P}\left\{{ }^{1} \mathrm{H}\right\}$ NMR spectroscopy, by $2 \mathrm{D}{ }^{1} \mathrm{H}-{ }^{1} \mathrm{H}$ COSY NMR spectroscopy (Figure S26 and S27), by mass spectrometry, and by ${ }^{31} \mathrm{P}\left\{{ }^{1} \mathrm{H}\right\}$ NMR spectroscopy of the corresponding ${ }^{15} \mathrm{~N}$-labeled isotopomer trans-8- $-{ }^{15} \mathrm{~N}$. The numbering scheme for the aliphatic ${ }^{1} \mathrm{H}$ resonances of trans8 is depicted in Figure S28. The formation of a four-membered metallacycle was indicated by the strong upfield shift of the diastereotopic platinum-bound methylene protons at $\delta 0.06\left(\mathrm{dt}, J_{\mathrm{HH}}=1.7,9.1 \mathrm{~Hz} ; J_{\mathrm{PtH}} \approx 100 \mathrm{~Hz}\right)$ and $\delta-0.34\left(\mathrm{td}, J_{\mathrm{HH}}=3.9,9.3 \mathrm{~Hz}\right.$; $\left.J_{\mathrm{PtH}} \approx 100 \mathrm{~Hz}\right)$ (Figure S29). Both the presence of an N-Pt bond and the trans arrangement of the amine and phosphine ligands of trans-8 was firmly established by the large ${ }^{31} \mathrm{P}_{-}{ }^{15} \mathrm{~N}$ coupling constant $\left(J_{\mathrm{PN}}=47 \mathrm{~Hz}\right)$ observed in the ${ }^{31} \mathrm{P}\left\{{ }^{1} \mathrm{H}\right\}$ NMR spectrum of trans $-8-{ }^{15} \mathrm{~N} .{ }^{\mathrm{S} 11}$

trans- $\mathrm{PtCl}_{2}\left(\mathrm{PPh}_{3}\right)\left[\eta^{1}-\mathrm{NHBnCH}_{2} \mathrm{C}\left(\mathrm{Ph}_{2}\right) \mathrm{CH}_{2} \mathrm{CH}=\mathrm{CH}_{2}\right] \quad$ (trans-5). A frozen suspension of 1 (5.4 mg, $0.017 \mathrm{mmol}), \mathrm{PhSiMe}_{3}\left(0.32 \mathrm{mg}, 2.1 \times 10^{-3} \mathrm{mmol}\right)$, and 4 (8.9 $\left.\mathrm{mg}, 8.4 \times 10^{-3} \mathrm{mmol}\right)$ in $\mathrm{CDCl}_{3}(1.16 \mathrm{~mL})$ at $-78^{\circ} \mathrm{C}$ was thawed briefly to form a yellow solution that was placed into the probe of an NMR spectrometer pre-cooled at $-20{ }^{\circ} \mathrm{C}$. ${ }^{1} \mathrm{H}$ NMR analysis of the resulting solution after 5 min revealed complete consumption of 4 to form trans -5 in $97 \pm 5 \%$ yield as was determined by integrating the olefinic resonances of trans-5 at $\delta$ 5.90-5.98 $\left(H_{5}\right), 5.09\left(H_{7}\right)$, and $4.98\left(H_{6}\right)$ relative to the methyl resonance of $\mathrm{PhSiMe}_{3}$ at $\delta 0.02$. Thermally unstable trans $-\mathbf{5}$ was characterized by 
spectroscopy without isolation. ${ }^{1} \mathrm{H}\left\{{ }^{31} \mathrm{P}\right\}$ NMR $\left(500 \mathrm{MHz},-20{ }^{\circ} \mathrm{C}\right): \delta \quad 6.84-7.82(\mathrm{~m}, 30$ H), 5.90-5.98 (m, $\left.1 \mathrm{H}, H_{5}\right), 5.09\left(\mathrm{~d}, J=16.9 \mathrm{~Hz}, 1 \mathrm{H}, H_{7}\right), 4.98\left(\mathrm{~d}, J=10.3 \mathrm{~Hz}, 1 \mathrm{H}, H_{6}\right)$, $4.57\left(\mathrm{dd}, J=8.0,12.5 \mathrm{~Hz}, 1 \mathrm{H}, H_{3}\right), 4.52\left(\mathrm{dd}, J=6.6,12.7 \mathrm{~Hz}, 1 \mathrm{H}, H_{l}\right), 4.13(\mathrm{dd}, J=$ 5.9, $\left.14.1 \mathrm{~Hz}, 1 \mathrm{H}, H_{4}\right), 3.81\left(\mathrm{t}, J=7.1 \mathrm{~Hz}, 1 \mathrm{H}, H_{2}\right), 3.44\left(\mathrm{dd}, J=7.3,12.8 \mathrm{~Hz}, 1 \mathrm{H}, H_{l}\right)$, 3.12-3.19 (m, $\left.2 \mathrm{H}, H_{3}+H_{4}\right) .{ }^{31} \mathrm{P}\left\{{ }^{1} \mathrm{H}\right\} \mathrm{NMR}\left(-20^{\circ} \mathrm{C}\right): \delta 5.20\left(\mathrm{~s}, J_{\mathrm{PtP}}=3566 \mathrm{~Hz}\right)$.

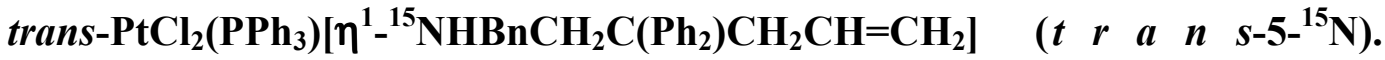
Reaction of $1-{ }^{15} \mathrm{~N}(6.9 \mathrm{mg}, 0.021 \mathrm{mmol})$ and $4(11.1 \mathrm{mg}, 0.011 \mathrm{mmol})$ in $\mathrm{CDCl}_{3}(1.00$ $\mathrm{mL}$ ) employing a procedure analogous to that used to synthesize unlabelled trans-5 formed trans-5- ${ }^{15} \mathrm{~N}$ as the exclusive product. Thermally sensitive trans $-5-{ }^{15} \mathrm{~N}$ was characterized in solution by ${ }^{31} \mathrm{P}$ NMR spectroscopy. ${ }^{31} \mathrm{P}\left\{{ }^{1} \mathrm{H}\right\}$ NMR $\left(-40{ }^{\circ} \mathrm{C}\right): \delta 5.20(\mathrm{~d}$, $\left.J_{\mathrm{NP}}=47 \mathrm{~Hz}, J_{\mathrm{PtP}}=3610 \mathrm{~Hz}\right)$.

$\operatorname{PtCl}_{2}\left(\mathrm{PPh}_{3}\right)\left(\mathrm{CH}_{2} \mathrm{CHCH}_{2} \mathrm{C}\left(\mathrm{Ph}_{2}\right) \mathrm{CH}_{2} \mathrm{NHBn}\right)$ (6). A frozen solution of trans-5 $(0.017 \mathrm{mmol}, 15 \mathrm{mM})$ in $\mathrm{CDCl}_{3}(1.16 \mathrm{~mL})$ at $-78{ }^{\circ} \mathrm{C}$ was warmed at $20{ }^{\circ} \mathrm{C}$ and monitored in $105 \mathrm{~s}$ increments by ${ }^{1} \mathrm{H}$ NMR spectroscopy for $1 \mathrm{~h}$. Disappearance of trans5 was analyzed by integrating the olefinic resonance of trans-5 at $\delta 4.98\left(H_{6}\right)$ relative to the methyl resonance of $\mathrm{PhSiMe}_{3}$ at $\delta$ 0.02. A linear plot of $\ln \left\{[\text { trans }-5]_{t} /[\text { trans }-5]_{0}\right\}$ versus time established the first order decay of trans -5 to $>2$ half lives (Figure S22). The tube was then heated at $40{ }^{\circ} \mathrm{C}$ for 30 min to form 6 in $95 \pm 5 \%$ as determined by integrating the benzylic resonance of 6 at $\delta 5.25$ relative to the methyl resonance of $\mathrm{PhSiMe}_{3}$ at $\delta 0.02$.

Isolation of 6. A solution of $1(25 \mathrm{mg}, 0.076 \mathrm{mmol})$ and $4(41 \mathrm{mg}, 0.039 \mathrm{mmol})$ in $\mathrm{CDCl}_{3}(3 \mathrm{~mL})$ was allowed to stand at room temperature for $4 \mathrm{~h}$. The solvent was evaporated under vacuum to give pure 6 (66 $\mathrm{mg}, 100 \%)$ as a tan solid. 
For 6: ${ }^{1} \mathrm{H}$ NMR $(500 \mathrm{MHz})$ : $10.11\left(\right.$ br s, $\left.1 \mathrm{H}, H_{2}\right), 7.82(\mathrm{dd}, J=7.5,11.3 \mathrm{~Hz}, 6$ H), 7.14-7.48 (m, $20 \mathrm{H}), 7.05$ (d, $J=7.7 \mathrm{~Hz}, 2 \mathrm{H}), 6.89$ (d, $J=7.2 \mathrm{~Hz}, 2 \mathrm{H}), 5.25$ (dd, $J$ $\left.=2.8,12.3 \mathrm{~Hz}, 1 \mathrm{H}, H_{3}\right), 3.75-3.88\left(\mathrm{~m}, 2 \mathrm{H}, H_{1}\right), 2.72\left(\mathrm{dd}, J=5.6,14.0 \mathrm{~Hz}, 1 \mathrm{H}, H_{4}\right)$, $2.62\left(\mathrm{dd}, J=10.0,12.8 \mathrm{~Hz}, 1 \mathrm{H}, H_{3}\right), 2.39-2.52\left(\mathrm{~m}, 1 \mathrm{H}, H_{5}\right), 2.08(\mathrm{dd}, J=11.2,13.6 \mathrm{~Hz}$, $\left.1 \mathrm{H}, H_{4}\right), 1.94\left(\mathrm{t}, J=11.1 \mathrm{~Hz}, 1 \mathrm{H}, H_{6}\right), 1.42\left(\mathrm{ddd}, J=3.6,6.4,12.0 \mathrm{~Hz}, 1 \mathrm{H}, H_{6}\right)$. ${ }^{31} \mathrm{P}\left\{{ }^{1} \mathrm{H}\right\} \mathrm{NMR}: \delta 14.19\left(\mathrm{~s}, J_{\mathrm{PtP}}=4844 \mathrm{~Hz}\right)$. ESIMS calcd (found) for $\mathrm{C}_{42} \mathrm{H}_{40} \mathrm{CINPPt}$ $\left(\mathrm{M}^{+}-\mathrm{Cl}\right) 820.3(820.2)$.

$\mathrm{PtCl}_{2}\left(\mathrm{PPh}_{3}\right)\left(\mathrm{CH}_{2} \mathrm{CHCH}_{2} \mathrm{C}\left(\mathrm{Ph}_{2}\right) \mathrm{CH}_{2}{ }^{15} \mathrm{NHBn}\right)\left(6-{ }^{15} \mathrm{~N}\right)$. A solution of trans-5${ }^{15} \mathrm{~N}(0.021 \mathrm{mmol}, 21 \mathrm{mM})$ in $\mathrm{CDCl}_{3}(1.00 \mathrm{~mL})$ was warmed at $25^{\circ} \mathrm{C}$ for $3 \mathrm{~h}$ to form 7 ${ }^{15} \mathrm{~N}$ as the exclusive product and was characterized without isolation by ${ }^{31} \mathrm{P}\left\{{ }^{1} \mathrm{H}\right\} \mathrm{NMR}$ spectroscopy. ${ }^{31} \mathrm{P}\left\{{ }^{1} \mathrm{H}\right\}$ NMR: $\delta 14.19\left(\mathrm{~s}, J_{\mathrm{PtP}}=4848 \mathrm{~Hz}\right)$.

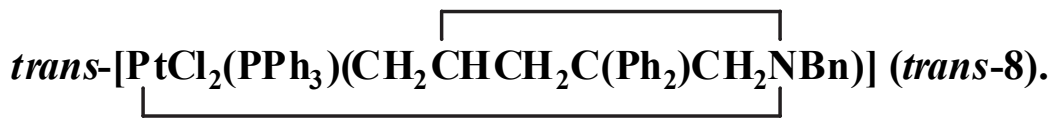

Diethylamine $(3.4$ $\mu \mathrm{L}, 0.033 \mathrm{mmol})$ was added to an NMR tube containing a solution of $6(0.33 \mathrm{mmol}, 33$ $\mathrm{mM})$ and $\mathrm{PhSiMe}_{3}\left(1 \mu \mathrm{L}, 6 \times 10^{-3} \mathrm{mmol}\right)$ in $\mathrm{CDCl}_{3}(1.00 \mathrm{~mL})$ and the tube was shaken briefly. ${ }^{1} \mathrm{H}$ NMR analysis of the resulting solution within 5 min of mixing revealed formation of trans-8 in $104 \pm 5 \%$ yield as was determined by integrating the olefinic resonance of trans $\mathbf{- 8}$ at $\delta 5.04\left(\mathrm{H}_{1} / \mathrm{H}_{2}\right)$ relative to the methyl resonance of $\mathrm{PhSiMe}_{3}$ at $\delta$ 0.02 .

Isolation of trans-8. A solution of $1(24.7 \mathrm{mg}, 0.075 \mathrm{mmol})$ and $4(39.8 \mathrm{mg}$, $0.038 \mathrm{mmol})$ in $\mathrm{CDCl}_{3}(3 \mathrm{~mL})$ was allowed to stand at room temperature for $2 \mathrm{~h}$, treated with diethylamine $(7.8 \mu \mathrm{L}, 0.075 \mathrm{mmol})$, and allowed to stand for an additional $10 \mathrm{~min}$. The resulting solution was diluted with EtOAc $(2 \mathrm{~mL})$ and $\mathrm{CHCl}_{3}(10 \mathrm{~mL})$, washed with 
water $(3 \times 10 \mathrm{~mL})$, and dried $\left(\mathrm{MgSO}_{4}\right)$. The solvent was evaporated under vacuum and the resulting residue was dissolved in $\mathrm{CHCl}_{3}(1 \mathrm{~mL})$. Hexanes $(10 \mathrm{~mL})$ were added and the resulting precipitate was filtered off and washed with hexanes $(10 \mathrm{~mL})$. The resulting solid was suspended in methanol $(1 \mathrm{~mL})$, sonicated for $5 \mathrm{~min}$, filtered, and dried under vacuum to give trans-8 (5 mg, 8\%) as an off-white solid.

For trans-8: ${ }^{1} \mathrm{H}$ NMR $\left(500 \mathrm{MHz}, \mathrm{CDCl}_{3}\right): \delta 7.79(\mathrm{~d}, J=7.0 \mathrm{~Hz}, 2 \mathrm{H}), 7.51(\mathrm{~d}$, $J=7.5 \mathrm{~Hz}, 2 \mathrm{H}), 7.17-7.44(\mathrm{~m}, 25 \mathrm{H}), 7.06(\mathrm{t}, J=7.1 \mathrm{~Hz}, 1 \mathrm{H}), 5.26-5.32\left(\mathrm{~m}, 1 \mathrm{H}, H_{4}\right)$, $5.04\left(\mathrm{dd}, J=4.0,12.5 \mathrm{~Hz}, 1 \mathrm{H}, H_{1} / H_{2}\right), 4.56\left(\mathrm{~d}, J=11.4 \mathrm{~Hz}, 1 \mathrm{H}, H_{1} / H_{2}\right), 3.85(\mathrm{dd}, J=$ 2.9, $\left.12.5 \mathrm{~Hz}, 1 \mathrm{H}, H_{1} / H_{2}\right), 3.35\left(\mathrm{dd}, J=8.3,11.8 \mathrm{~Hz}, 1 \mathrm{H}, H_{1} / H_{2}\right), 3.04(\mathrm{dd}, J=7.8,13.3$ $\left.\mathrm{Hz}, 1 \mathrm{H}, H_{3}\right), 2.48\left(\mathrm{dd}, J=7.5,13.5 \mathrm{~Hz}, 1 \mathrm{H}, H_{3}\right), 0.06\left(\mathrm{dt}, J=1.7,9.1 \mathrm{~Hz}, 1 \mathrm{H}, H_{5}\right)$, $-0.34\left(\mathrm{td}, J=3.9,9.3 \mathrm{~Hz}, 1 \mathrm{H}, H_{5}\right) .{ }^{31} \mathrm{P}\left\{{ }^{1} \mathrm{H}\right\} \mathrm{NMR}: \delta 8.61\left(\mathrm{~s}, J_{\mathrm{PtP}}=4402 \mathrm{~Hz}\right)$. ESIMS calcd (found) for $\mathrm{C}_{42} \mathrm{H}_{39} \mathrm{NPPt}\left(\mathrm{M}^{+}-\mathrm{Cl}\right)$ : 783.2 (783.3).

\section{trans-[PtCl $\left.{ }_{2}\left(\mathrm{PPh}_{3}\right)\left(\mathrm{CH}_{2} \mathrm{CHCH}_{2} \mathrm{C}\left(\mathrm{Ph}_{2}\right) \mathrm{CH}_{2}{ }^{15} \mathrm{NBn}\right)\right]\left(\right.$ trans $\left.-8-{ }^{15} \mathrm{~N}\right)$.}

Reaction of

diethylamine $(3.4 \mu \mathrm{L}, 0.033 \mathrm{mmol})$ with $6{ }^{15} \mathrm{~N}(0.33 \mathrm{mmol}, 33 \mathrm{mM})$ employing a procedure similar to that used to synthesize trans-8 formed $\operatorname{trans}-\mathbf{8}-{ }^{15} \mathrm{~N}$ as the exclusive product. trans $-8-{ }^{15} \mathrm{~N}$ was analyzed without isolation by ${ }^{31} \mathrm{P}$ NMR spectroscopy. ${ }^{31} \mathrm{P}\left\{{ }^{1} \mathrm{H}\right\}$ NMR: $\delta 8.61\left(\mathrm{~d}, J_{\mathrm{NP}}=47.4 \mathrm{~Hz}, J_{\mathrm{PtP}}=4424 \mathrm{~Hz}\right)$.

Conversion of 6 to 3 in the Presence of 7. Benzyl-4-pentenylamine (7) (26.5 $\mathrm{mg}, 151 \mathrm{mmol})$ was added to a solution of $6(27.2 \mathrm{mg}, 0.032 \mathrm{mmol})$ and $\mathrm{PhSiMe}_{3}(1 \mu \mathrm{L}$, $\left.6 \times 10^{-3} \mathrm{mmol}\right)$ in dioxane- $d_{8}(1.00 \mathrm{~mL}) .{ }^{1} \mathrm{H} \mathrm{NMR}$ analysis of the resulting solution 5 min after mixing revealed complete consumption of $\mathbf{6}$ to form heterobicyclic amine complex trans $\mathbf{- 8}$. The relative concentration of trans $\mathbf{- 8}$ was determined by integrating the 
$\mathrm{H}_{1} / \mathrm{H}_{2}$ resonance of trans-8 (Figure S28) at $\delta 4.56$ relative to the methyl resonance of $\mathrm{PhSiMe}_{3}$ at $\delta 0.02$. The solution was then heated at $120{ }^{\circ} \mathrm{C}$ for $16 \mathrm{~h} .{ }^{1} \mathrm{H}$ NMR analysis of the resulting solution revealed formation of 3 in $101 \pm 5 \%$ yield by integrating the methyl doublet of $\mathbf{3}$ at $\delta 1.21$ relative to the methyl resonance of $\mathrm{PhSiMe}_{3}$.

Reaction of 3 with 4 . A solution of $3(8.4 \mathrm{mg}, 0.026 \mathrm{mmol})$ and $4(13.7 \mathrm{mg}$, $0.013 \mathrm{mmol})$ in $\mathrm{CDCl}_{3}(1.0 \mathrm{~mL})$ was allowed to stand at room temperature for $1 \mathrm{~h}$. ${ }^{31} \mathrm{P}\left\{{ }^{1} \mathrm{H}\right\}$ NMR analysis revealed $37 \%$ consumption to form a mixture of seven platinum complexes with resonances at $\delta 15.36,7.34(\mathrm{~s}, J=2598 \mathrm{~Hz}), 4.87,4.50,4.31,2.69$, -1.72 that accounted for $37 \%$ of the phosphine ligand from 4 . The four resonances in region from $\delta 2-5$ are consistent with a $\mathrm{PPh}_{3}$ ligand bonded to a platinum amine complex. 